\title{
ß-Amino Phosphine Mn Catalysts for 1,4-Transfer Hydrogenation of Chalcones and Allylic Alcohol Isomerization
}

Vipulan Vigneswaran†, Samantha N. MacMillan§, and David C. Lacy ${ }^{\star *}$

Department of Chemistry, University at Buffalo, State University of New York, Amherst, New York 14260, United States.

sDepartment of Chemistry and Chemical Biology, Cornell University, Ithaca, New York 14853, United States.

E-mail: DCLacy@buffalo.edu

\section{Contents}

Page

General considerations

Ligand Synthesis and Characterization

S2

Complex Synthesis and Characterization

$\mathrm{S} 2-\mathrm{S}_{3}$

Substrate Scope

$\mathrm{S} 3-\mathrm{S} 6$

References

$\mathrm{S} 7-\mathrm{S} 22$

$\mathrm{S} 22$ 


\section{General Considerations}

Chemicals were obtained from commercial vendors unless noted. All manipulations of oxygen sensitive compounds were performed under an argon atmosphere with standard Schlenk techniques or under nitrogen in a VAC Atmosphere Genesis Glovebox. Anhydrous organic solvents were sparged with argon and purified using a Pure Process Technology solvent purification system and were stored over $3 \AA$ molecular sieves before use. Deuterated solvents were degassed via three freeze-pump-thaw cycles and stored over sieves in a glovebox.

${ }^{1} \mathrm{H}$ NMR spectra were recorded on a Varian Mercury-300 or Varian Inova-400 MHz spectrometer referenced to residual solvent proton signals. ${ }^{31} \mathrm{P}$ NMR spectra were recorded on a Varian Mercury-300 $\mathrm{MHz}$ spectrometer referenced to an external $\mathrm{H}_{3} \mathrm{PO}_{4}(85 \%)$ standard. Transmission ATR-FTIR spectra were collected using a Bruker Alpha IR spectrometer with ALPHA-P Platinum ATR module (diamond crystal) under an argon atmosphere inside of a VAC Atmospheres Omni Glovebox. Ketones were purchased from commercial vendors and their respective chalcones were synthesized from a literature procedure. ${ }^{1}$ Allyl alcohols were synthesized from a literature procedure from their respective enones. ${ }^{2}$ Bishydroxyethyloxamide ligand and 2-((di-tert-butylphosphine)methyl)pyridine were synthesized following literature procedures.3,4 2-aminomethylpyridine, ethylene diamine, and 1,2-diphenylethylenediamine were obtained from commercial sources.

\section{Crystallographic Methods}

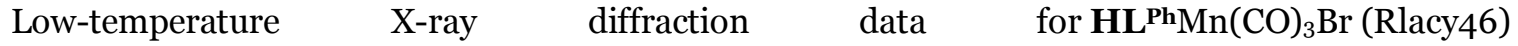
and $\mathbf{E M n}(\mathrm{CO})_{3} \mathrm{Br}$ (Rlacy44) were collected on a Rigaku XtaLAB Synergy diffractometer coupled to a Rigaku Hypix detector with $\mathrm{Cu} \mathrm{Ka}$ radiation $(\lambda=1.54184 \AA)$ from a PhotonJet micro-focus X-ray source at $100 \mathrm{~K}$. The diffraction images were processed and scaled using the CrysAlisPro software. 5 The structures were solved through intrinsic phasing using SHELXT ${ }^{6}$ and refined against F2 on all data by full-matrix least squares with SHELXL7 following established refinement strategies. ${ }^{8}$ All non-hydrogen atoms were refined anisotropically. All hydrogen atoms bound to carbon were included in the model at geometrically calculated positions and refined using a riding model. Hydrogen atoms bound to nitrogen were located in the difference Fourier synthesis and subsequently refined semi-freely with the help of distance restraints. The isotropic displacement parameters of all hydrogen atoms were fixed to 1.2 times the $\mathrm{U}_{\text {eq }}$ value of the atoms they are linked to (1.5 times for methyl groups). The molecular structure of $\mathbf{H L} \mathbf{L h}^{\mathbf{P h}} \mathrm{Mn}(\mathrm{CO}){ }_{3} \mathrm{Br}$ contained two independent molecules, both of which have the same configuration. The molecular structure of $\mathbf{E M n}(\mathrm{CO})_{3} \mathrm{Br}$ contains disorder of the bromine and one of the $\mathrm{CO}$ ligands, which was modeled satisfactorily over the two positions.

\section{Ligand Synthesis}

(S)-2-(diphenylphosphanyl)-1-phenylethan-1-amine (HL $\left.{ }^{\mathrm{Ph}}\right)$. The ligand was synthesized following a modified literature report. 9 In a glovebox, a $100 \mathrm{~mL}$ Schlenk flask, equipped with a stirbar, was charged with $20 \mathrm{~mL}$ of dry, degassed toluene. (S)-4-phenyl-2-oxazolidinone (326 mg, $2.00 \mathrm{mmol}, 1$ eq.) was added and allowed to stir at room temperature for 15 minutes to form a well dispersed suspension. To this suspension, diphenylphosphine was added in one portion by Pasteur pipette (633 mg, $3.40 \mathrm{mmol}$, 1.7 eq.). This mixture was allowed to stir for a further 30 minutes. The flask was subsequently sealed and brought out of the glovebox, brought under positive argon flow, and then placed in a mineral oil bath to stir at room temperature. Trifluoromethanesulfonic acid ( $480 \mu \mathrm{L}, 5.4 \mathrm{mmol}$. 2.7 eq.) was added via glass syringe in one portion through a rubber septum. It was noted that thin, wispy, fumes appear within the flask at this time. Once the fumes had subsided, the rubber septum was removed, and a cold finger was equipped to the flask under a strong flow of argon. The cold finger was connected to a temperature-controlled circulator maintained at $15{ }^{\circ} \mathrm{C}$. The mixture was then brought to reflux for a period of 24 hours. As the reaction reached reflux temperature it became more homogeneous.

Upon conclusion of heating, the mixture was brought back to room temperature and the cold finger was removed and replaced with a rubber septum under positive argon flow. To this mixture, degassed, saturated $\mathrm{K}_{2} \mathrm{CO}_{3}$ aqueous solution (10 mL) was added via cannula. The reaction was allowed to quench for a period of 30 minutes and then the organics were extracted with the aid of additional degassed diethyl ether $(3 \times 20 \mathrm{~mL})$ directly onto anhydrous sodium sulfate. The dried organic extracts were decanted with cannula transfer into a new flask and solvent was removed in vacuo to yield an oil or glassy solid. In a glovebox, the oil was dissolved in petroleum ether $(10 \mathrm{~mL})$ and stored in a freezer at $-35{ }^{\circ} \mathrm{C}$ to induce precipitation. The solid was isolated on a glass fritted funnel, washed with petroleum ether, and dried with vacuum (523 mg, $85 \%$ yield). The spectral features of the product were in agreement with literature 
characterization values. ${ }^{1} \mathrm{H}$ NMR $(300 \mathrm{MHz}$ ) $\delta 7.73-6.95(\mathrm{~m}, 14 \mathrm{H}), 4.00$ (ddd, $J=9.3,7.4,4.8 \mathrm{~Hz}, 1 \mathrm{H}$ ), 2.53 (ddd, $J=13.6,4.8,2.3 \mathrm{~Hz}, 1 \mathrm{H}$ ), 2.41 (ddd, $J=13.7,9.2,2.2 \mathrm{~Hz}, 1 \mathrm{H}$ ). Characterization Figures S1-S2

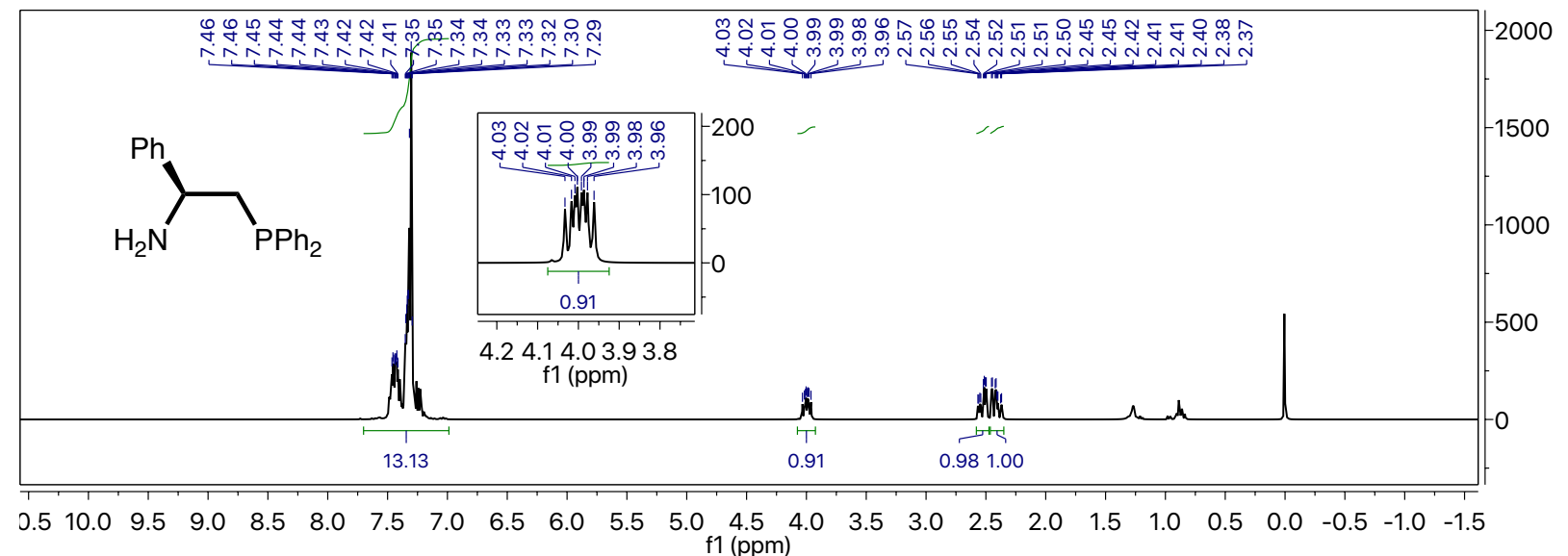

Figure S1. ${ }^{1} \mathrm{H}$ NMR spectrum of $\mathbf{H L}^{\mathbf{P h}}$ in $\mathrm{CDCl}_{3}\left({ }^{*}\right)$.

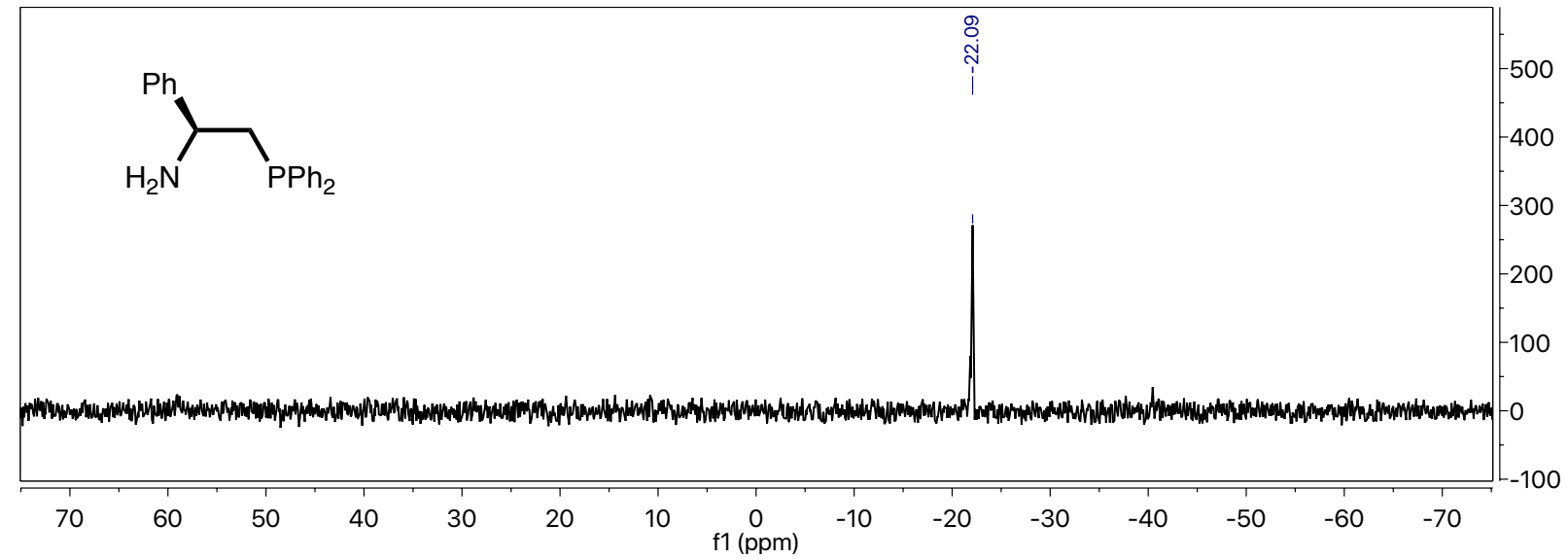

Figure S2. ${ }^{11} \mathrm{P}\{1 \mathrm{H}\}$ NMR Spectrum of ligand in $\mathrm{CDCl}_{3}$.

\section{Complex Synthesis}

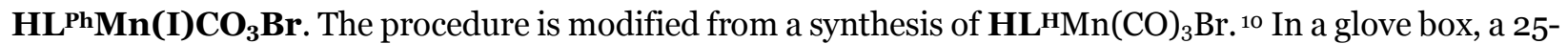
$\mathrm{mL}$ thick-walled glass Schlenk bomb, equipped with a stir bar, was charged with degassed, anhydrous toluene ( $4 \mathrm{~mL}$ ) and the ligand $\mathbf{H L}^{\mathbf{P h}}(198 \mathrm{mg}, 0.648 \mathrm{mmol})$. The mixture was stirred at room temperature for 5 minutes. Bromopentacarbonylmanganese(I) $(178 \mathrm{mg}$, $0.648 \mathrm{mmol})$ was then added in one portion. The reaction bomb was then sealed and stirred at room temperature for 5 minutes. Carbon monoxide liberation was observed as a stream of microbubbles formed from the solution. The mixture is then removed from the glovebox and heated at $100^{\circ} \mathrm{C}$ for 12 hours. The mixture changes color from orange to a golden yellow-orange color over this time. Upon conclusion of heating and gradual cooling to room temperature, the reaction bomb is brought back into the glovebox and the mixture is transferred to a tared scintillation vial. The solvent was removed in vacuo and the resulting residue was dissolved in a minimal amount of THF $(\sim 2.5 \mathrm{~mL})$. The concentrated THF solution was then layered under petroleum ether that yielded yellow-orange crystals $(283 \mathrm{mg}, 83.4 \%) .{ }^{1} \mathrm{H} \mathrm{NMR}\left(300 \mathrm{MHz}, \mathrm{CD}_{2} \mathrm{Cl}_{2}\right) \delta 7.84\left(t, \mathrm{~J}=6 \mathrm{~Hz}, 2 \mathrm{H}, \mathrm{PC}_{6} \mathrm{H}_{6}-m-H\right)$, $7.69\left(t, \mathrm{~J}=6 \mathrm{~Hz}, 2 \mathrm{H}, \mathrm{PC}_{6} \mathrm{H}_{6}-m-H\right)$ 7.52-7.41 $\left(m, 11 \mathrm{H}, \mathrm{Ar}-H, \mathrm{PC}_{6} \mathrm{H}_{6}-\mathrm{o}-H\right.$, and $\left.\mathrm{PC}_{6} \mathrm{H}_{6}-p-H\right), 3.99(s, 1 \mathrm{H}, \mathrm{N}-$ $H \mathrm{H}), 3.77(s, 1 \mathrm{H}, \mathrm{N}-\mathrm{H} H), 3.19\left(t, \mathrm{~J}=14 \mathrm{~Hz}, 2 \mathrm{H}, \mathrm{C}-H_{2}\right), 2.76(t, \mathrm{~J}=14 \mathrm{~Hz}, 1 \mathrm{H}, \mathrm{C}-H) .{ }^{3} \mathrm{P}\left\{{ }^{1} \mathrm{H}\right\} \mathrm{NMR}(121 \mathrm{MHz}$, $\mathrm{CDCl}_{3}$ ): 53.8 ppm. FTIR-ATR: 2017, $1935,1901 \mathrm{~cm}^{-1}$. Anal. Calcd. for $\mathrm{C}_{23} \mathrm{H}_{20} \mathrm{NO}_{3} \mathrm{MnBr}$ : C, 52.70\%; $\mathrm{H}$, $3.85 \%$; N, 2.67\%. Anal. Found for $\mathrm{C}_{23} \mathrm{H}_{20} \mathrm{NO}_{3} \mathrm{MnBr}$ : C, 52.67\%; H, 4.01\%; N, 2.63\%. Characterization Figures $\mathrm{S}_{3}-\mathrm{S}_{5}$ 


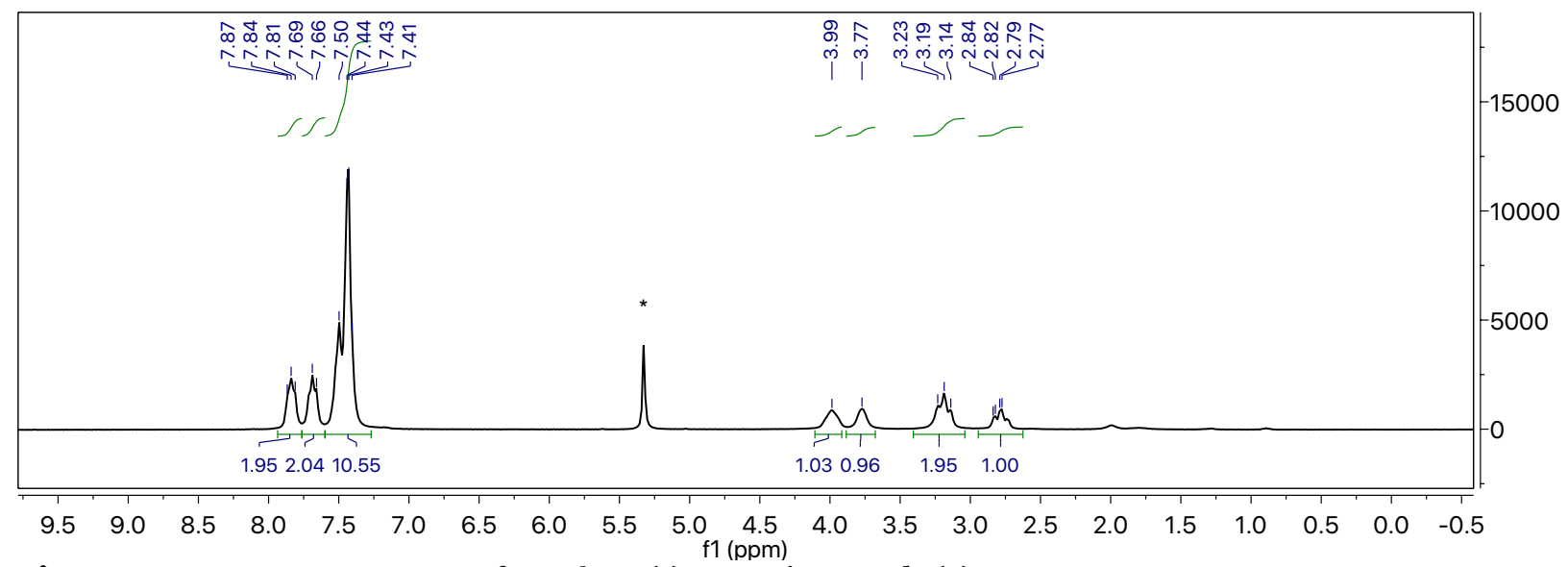

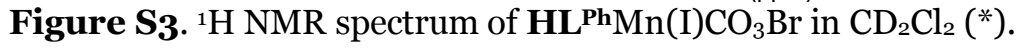

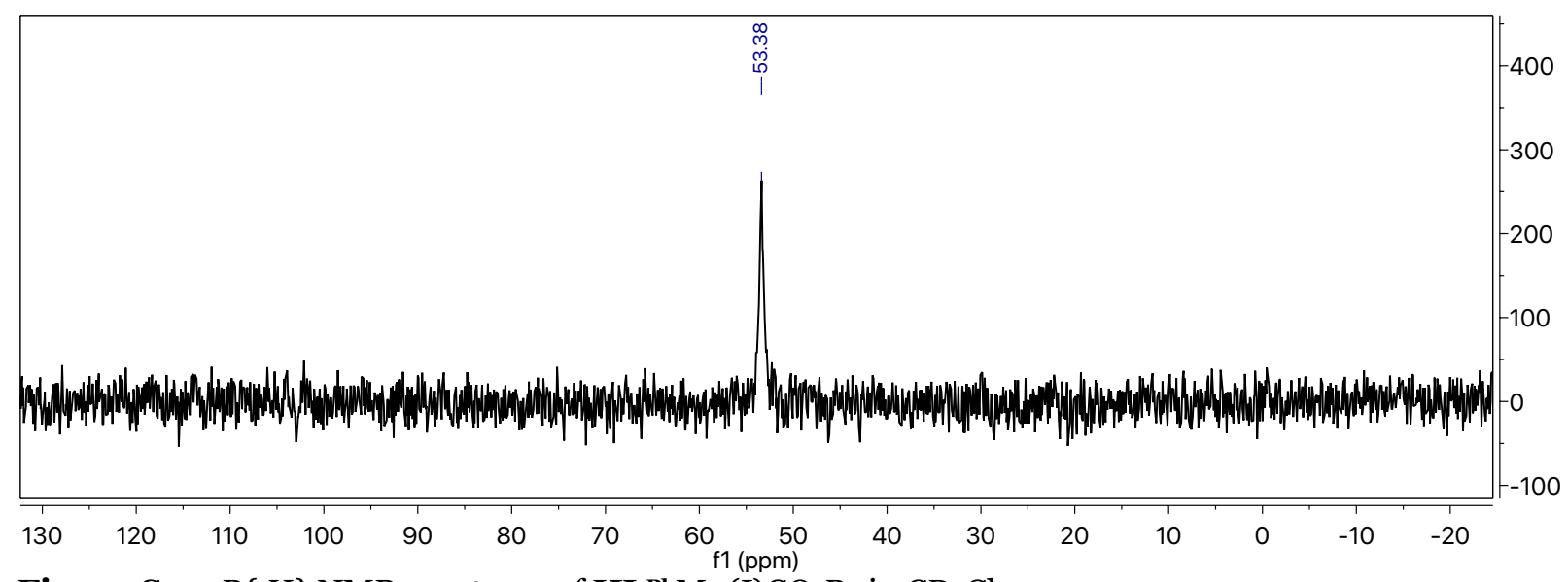

Figure S4. ${ }^{31} \mathrm{P}\left\{{ }^{1} \mathrm{H}\right\}$ NMR spectrum of $\mathbf{H L}^{\mathrm{Ph}} \mathrm{Mn}(\mathrm{I}) \mathrm{CO}_{3} \mathrm{Br}$ in $\mathrm{CD}_{2} \mathrm{Cl}_{2}$.

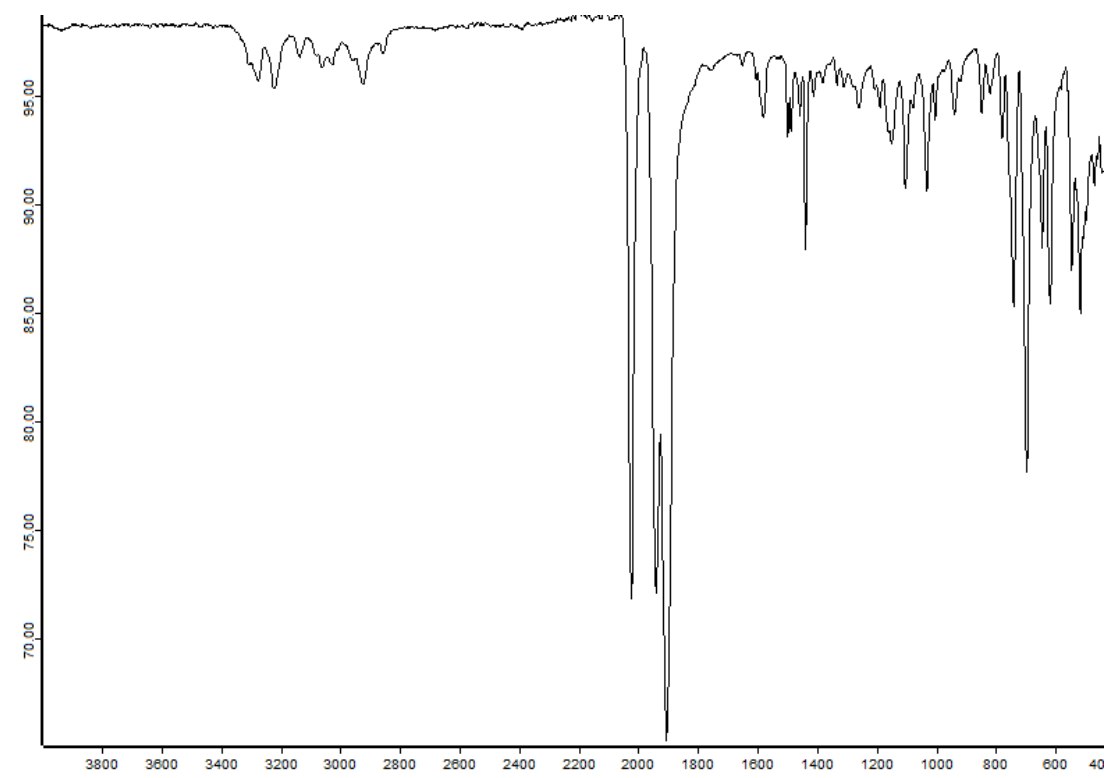

Figure S5. FTIR-ATR spectrum of $\mathbf{H L}^{\mathrm{Ph}} \mathrm{Mn}(\mathrm{I}) \mathrm{CO}_{3} \mathrm{Br}$ 
$\mathbf{L}^{\mathbf{P h}} \mathbf{M n}(\mathbf{I}) \mathbf{C O}_{3}$. Solutions containing the complex tentatively assigned as $\mathbf{L}^{\mathbf{P h}} \mathbf{M n}(\mathrm{I}) \mathrm{CO}_{3}$ was generated by one of two methods:

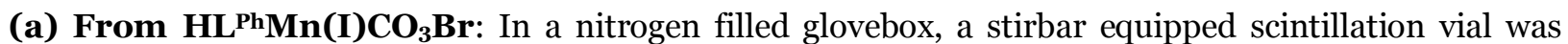

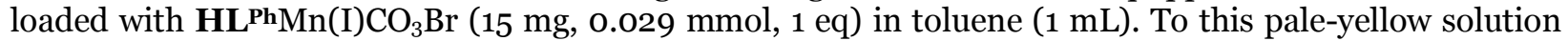
$\mathrm{KO}^{t} \mathrm{Bu}(3 \mathrm{mg}$, $0.03 \mathrm{mmol}, 1 \mathrm{eq}$; or $6 \mathrm{mg}, 0.06 \mathrm{mmol}, 2 \mathrm{eq}$.) was added in one portion and allowed to stir for 30 minutes, which induced a color change to amber ( 1 eq. $\mathrm{KO}^{t} \mathrm{Bu}$ ) or red (2 eq. $\mathrm{KO}^{t} \mathrm{Bu}$ ). A $450 \mu \mathrm{L}$ aliquot of the resulting homogeneous solution was transferred to a J-Young tube and a ${ }^{31} \mathrm{P}\left\{{ }^{1} \mathrm{H}\right\}$ NMR spectrum was collected. The mixture with two equivalents of $\mathrm{KO} t \mathrm{Bu}$ converted quantitatively a new species at ${ }^{31} \mathrm{P}\left\{{ }^{1} \mathrm{H}\right\}$ $\mathrm{NMR}=72.8 \mathrm{ppm}$. The tube with one equivalent of $\mathrm{KO}^{t} \mathrm{Bu}$ contained mostly unreacted starting material, but after heating over night at $100{ }^{\circ} \mathrm{C}$ gave rise to a $\sim 1: 1$ mixture of $\mathbf{H L}^{\mathrm{Ph}} \mathrm{Mn}(\mathrm{I}) \mathrm{CO}_{3} \mathrm{Br}$ and the same species a ${ }^{31} \mathrm{P}\left\{{ }^{1} \mathrm{H}\right\}$ NMR $72.8 \mathrm{ppm}$, which is tentatively assigned as $\mathbf{L}^{\mathrm{Ph}} \mathbf{M n}(\mathbf{I}) \mathbf{C O}_{3}$ (Figure S6a). An ATR-FTIR spectrum of the red material from 2 eq. $\mathrm{KO}^{t} \mathrm{Bu}$ has features associated with a facial tricarbonyl $(1994,1905$, $\left.1821 \mathrm{~cm}^{-1}\right)$.
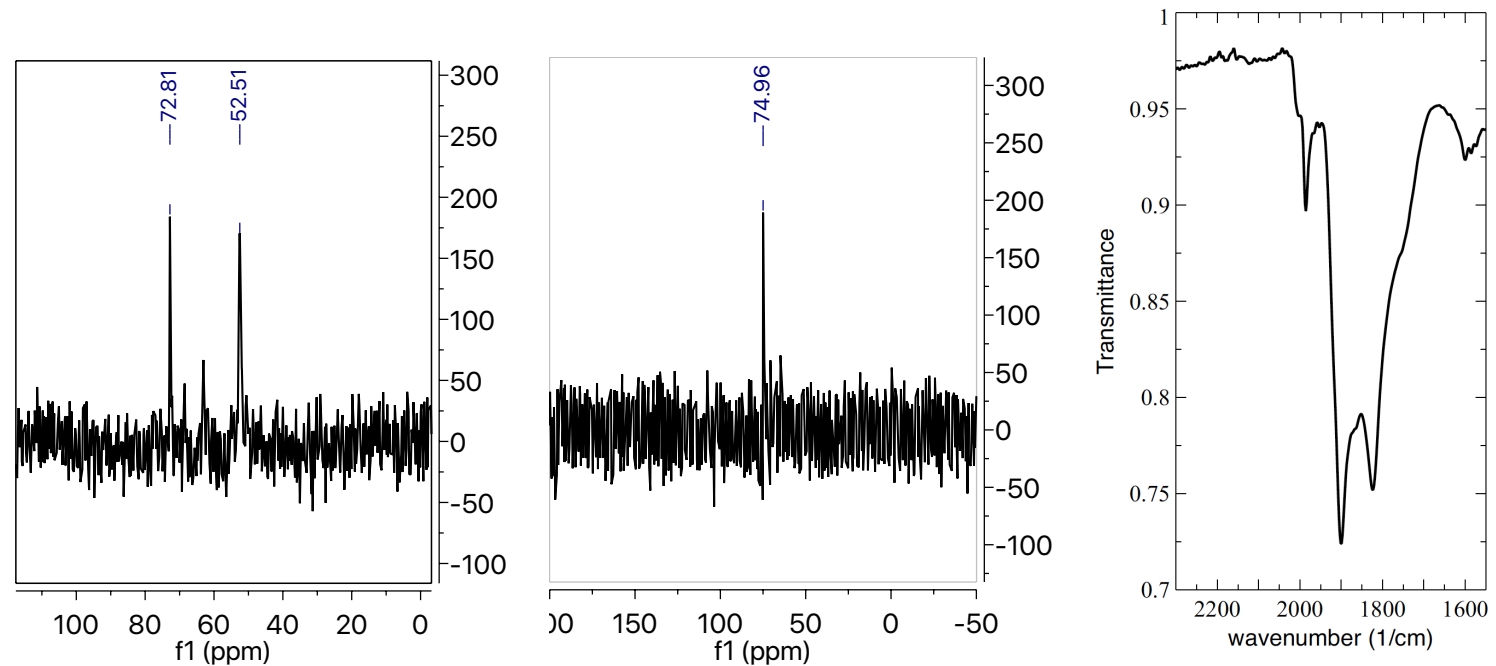

Figure S6a. ${ }^{1} \mathrm{P}\left\{{ }_{1} \mathrm{H}\right\}$ NMR spectrum (left) from overnight heating of a 1:1 mixture $\mathbf{H L}^{\mathrm{Ph}} \mathrm{Mn}(\mathrm{I}) \mathrm{CO}_{3} \mathrm{Br}$ and $\mathrm{KO}^{t} \mathrm{Bu}$ in toluene and (middle) mixing a 1:2 mixture of $\mathbf{H L}^{\mathrm{Ph}} \mathrm{Mn}(\mathrm{I}) \mathrm{CO}_{3} \mathrm{Br}$ and $\mathrm{KO}{ }^{t} \mathrm{Bu}$ in toluene. (right) ATR-FTIR spectrum of the red species.

(b) From HLPh and MeMn(CO) $)_{5}$ : In a nitrogen filled glovebox, toluene stock solutions of methyl manganese pentacarbonyl (60.5 $\mathrm{mM}$ ) and $\mathbf{H L}^{\mathrm{Ph}}$ were prepared (60.5 $\left.\mathrm{mM}\right)$. To a J-Young NMR tube, 275 $\mu \mathrm{L}$ of both stock solutions were added and slowly heated to $120^{\circ} \mathrm{C}$. The solution was initially clear and colorless and over time became pale yellow and later amber. . While pale yellow two ${ }^{3} \mathrm{P}\left\{{ }^{1} \mathrm{H}\right\}$ NMR peaks were observed (Figure S6b): 74.0 (s) and 65.0 (s) ppm. As the mixture was heated over a period of 18 hours, the mixture became increasingly darker in color from yellow to amber. The amber solution displayed three ${ }^{31} \mathrm{P}\left\{{ }^{1} \mathrm{H}\right\}$ NMR peaks (Figure S6b): 78.1 (s), 74.0 (s major) and $71.2(\mathrm{~s})$.
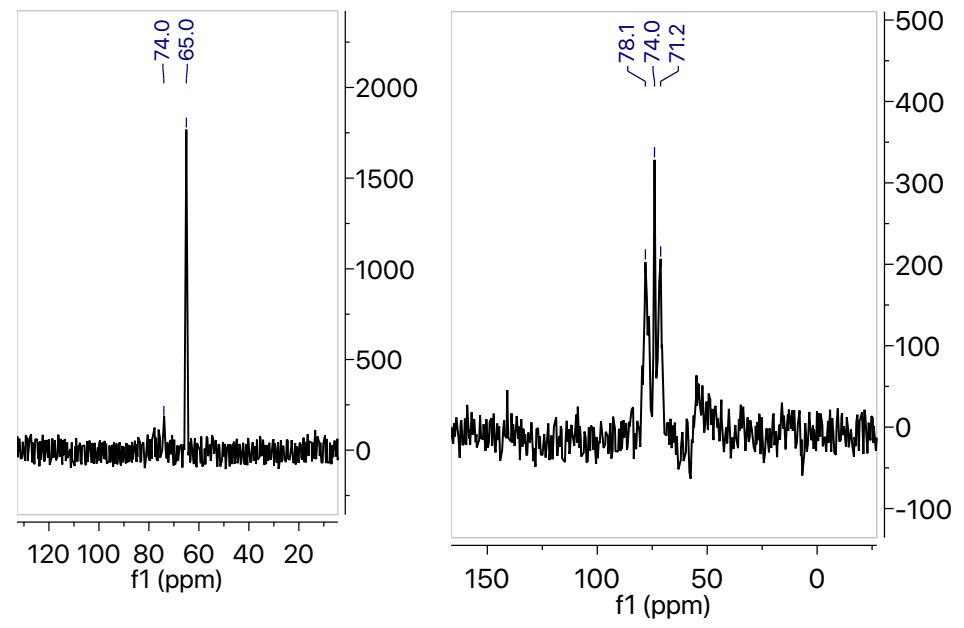

Figure S6b. ${ }^{31} \mathrm{P}\{1 \mathrm{H}\} \quad \mathrm{NMR}$ spectrum from the mixture of $\mathbf{H L}^{\text {Ph }}$ and $\mathrm{MeMn}(\mathrm{CO})_{5}$ in toluene at $120{ }^{\circ} \mathrm{C}$ after 3 hours (left) and after 18 hours (right). 
PicPtBuMn(I)CO $\mathbf{C O}_{3} \mathbf{B r}$. This complex was generated by the same procedure as $\mathbf{H L}^{\mathbf{P h}} \mathbf{M n}(\mathbf{I}) \mathbf{C O}_{3} \mathbf{B r}$, except using 2-((di-tert-butylphosphine)methyl)pyridine $(86 \mathrm{mg}, 0.36 \mathrm{mmol}, 1 \mathrm{eq})$ as the ligand. Crystals were grown from layering the crude reaction residue in THF under petroleum ether $(23 \mathrm{mg}, 15 \%$ crystalline yield). ${ }^{1} \mathrm{H}$ NMR (300 MHz, $\left.\mathrm{CD}_{2} \mathrm{Cl}_{2}\right) \delta 9.06\left(\mathrm{~d}, J=5.7 \mathrm{~Hz}, 1 \mathrm{H}, \mathrm{Ar}-H_{A}\right), 7.73\left(\mathrm{t}, J=7.6 \mathrm{~Hz}, 1 \mathrm{H}, \mathrm{Ar}-H_{C}\right), 7.44$ $\left(\mathrm{d}, J=7.8 \mathrm{~Hz}, 1 \mathrm{H}, \mathrm{Ar}-H_{D}\right), 7.22\left(\mathrm{t}, J=6.7 \mathrm{~Hz}, 1 \mathrm{H}, \mathrm{Ar}-H_{B}\right), 3.87(\mathrm{dd}, J=16.5,8.1 \mathrm{~Hz}, 1 \mathrm{H}, \mathrm{Ar}-\mathrm{CH} H), 3.47$ (dd, $J=16.4,9.0 \mathrm{~Hz}, 1 \mathrm{H}, \mathrm{Ar}-\mathrm{CHH}), 1.44\left(\mathrm{~d}, J=12.8 \mathrm{~Hz}, 9 \mathrm{H}, \mathrm{P}-\mathrm{C}-\left(\mathrm{CH}_{3}\right)\right), 1.36\left(\mathrm{~d}, J=12.5 \mathrm{~Hz}, 9 \mathrm{H}, \mathrm{P}-\mathrm{C}-\left(\mathrm{CH}_{3}\right)\right)$. ${ }^{31} \mathrm{P}\left\{{ }^{1} \mathrm{H}\right\}$ NMR (121 MHz, $\mathrm{CDCl}_{3}$ ): $88.2 \mathrm{ppm}$. HRMS (FT-ICR-MS): [M-Br] = 376.0857571. Calculated: 376.08743. Characterization Figure S7-S8

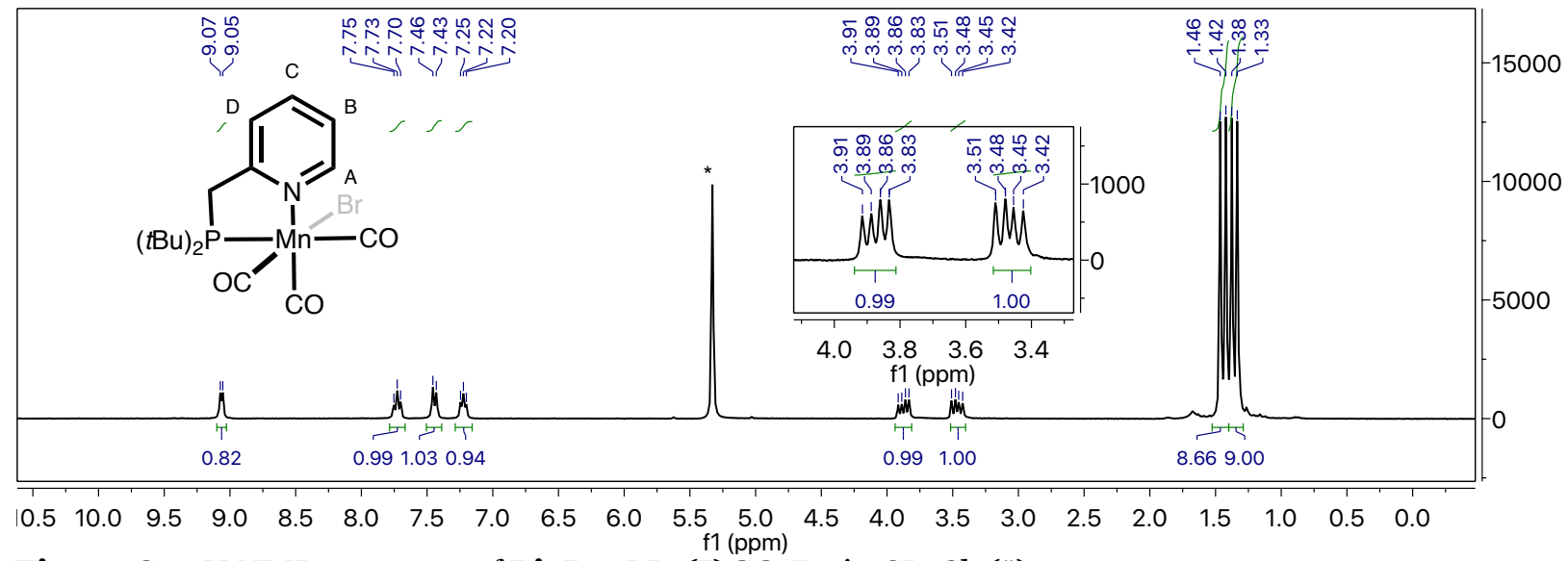

Figure S7. ${ }^{1} \mathrm{H}$ NMR spectrum of PicPtBu $\mathbf{M n}(\mathbf{I}) \mathbf{C O}_{3} \mathbf{B r}$ in $\mathrm{CD}_{2} \mathrm{Cl}_{2}\left({ }^{*}\right)$.

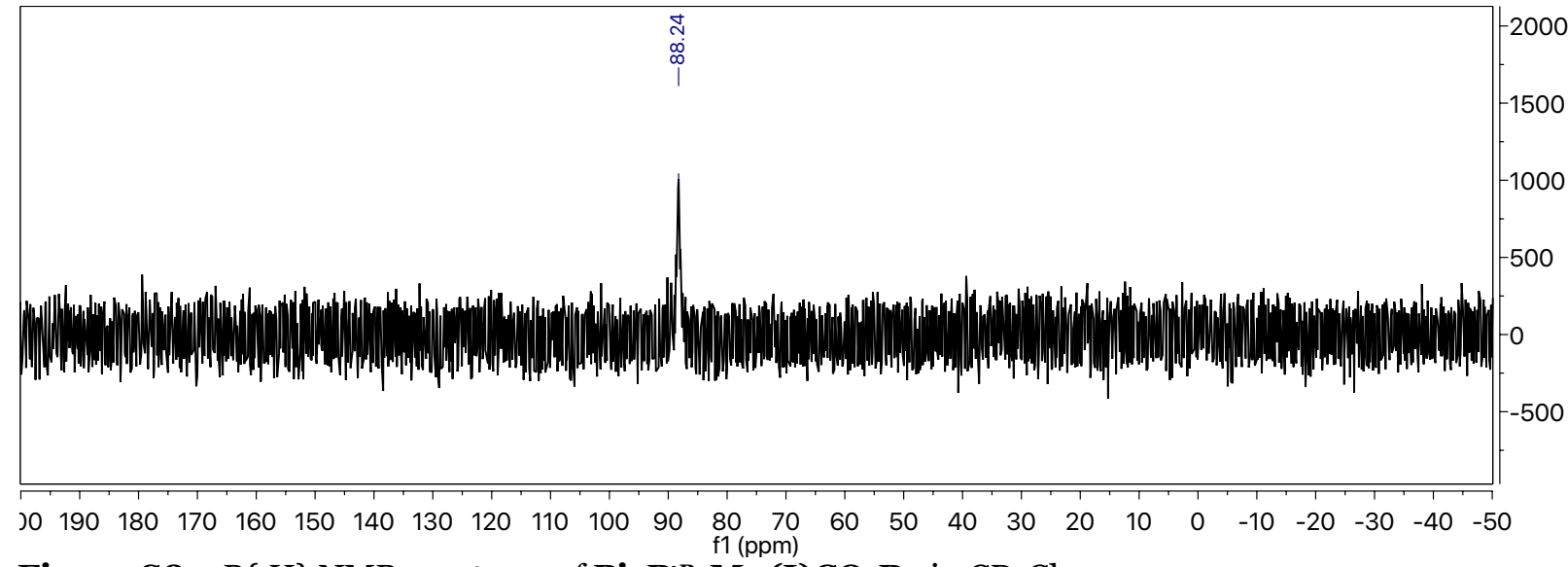

Figure S8. ${ }^{31} \mathrm{P}\left\{{ }^{1} \mathrm{H}\right\}$ NMR spectrum of PicPtBu Mn(I)CO ${ }_{3} \mathbf{B r}$ in $\mathrm{CD}_{2} \mathrm{Cl}_{2}$. 


\section{Substrate Scope}

\section{General Procedure}

Ketone transfer hydrogenation: Under an inert atmosphere, a $50 \mathrm{~mL}$ Schlenk flask or Schlenk bomb equipped with a small stir bar was charged with the ketone $(0.19 \mathrm{mmol})$ followed by $0.5 \mathrm{~mL}$ of degassed 2propanol and allowed to stir for 2 minutes. $1 \mathrm{~mL}$ of a fresh stock solution (0.96 mM) of $\mathbf{H L}^{\mathbf{P h}} \mathrm{MnCO}_{3} \mathrm{Br}$ in 2-propanol (0.5 mol\%) was added to the reaction mixture and allowed to stir for 10 minutes. $1 \mathrm{~mL}$ of a stock solution $(1.9 \mathrm{mM})$ of $\mathrm{KO}^{t} \mathrm{Bu}$ in 2-propanol $(1 \mathrm{~mol} \%)$ was added thereafter. The mixture was then heated at $60^{\circ} \mathrm{C}$, with a reflux condenser attached (or sealed), for a period of 3 hours under Argon; particular care must be taken to exclude oxygen. After the reaction is complete, the mixture was exposed to the atmosphere and the solution was passed through a short 2-inch silica plug (pipette). 2-propanol was removed using a rotary evaporator to obtain pure product. The isolated \% yields and ${ }^{1} \mathrm{H}-$ and ${ }^{13 \mathrm{C}}$-NMR spectra of isolated compounds are provided below. Incomplete conversions or crude materials were further purified by column chromatography, using EtOAc:Hex (5:95) unless otherwise mentioned.

Chalcones and $\alpha, \beta$-unsaturated compound transfer hydrogenation: The same procedure as above was used except the substrate amount was doubled (same concentration) and the reaction mixture was heated at $56^{\circ} \mathrm{C}$.

Allylic alcohol isomerization conditions: Under an inert atmosphere of nitrogen, in a glovebox, a thickwalled glass Schlenk bomb equipped with a stirbar was charged with toluene (4 mL) and $\mathbf{H L}^{\mathbf{P h}} \mathrm{MnCO}_{3} \mathrm{Br}$ ( $5.5 \mathrm{mg}, 0.010 \mathrm{mmol}, 1$ eq., $2 \mathrm{~mol} \%$ relative to substrate). The mixture was stirred until no more solids were observed. To this mixture, the allyl alcohol $(0.5 \mathrm{mmol})$ was added in one portion and stirred until homogeneous. Finally, $\mathrm{KO}^{t} \mathrm{Bu}(2.2 \mathrm{mg}$, $0.020 \mathrm{mmol}, 2 \mathrm{eq} ., 4 \mathrm{~mol} \%$ relative to substrate) was added to the reaction mixture. The vessel was then sealed and brought outside of the glovebox and heated to $110{ }^{\circ} \mathrm{C}$ for a period of 12 hours. The mixture was then allowed to return to room temperature and passed over a 1-inch celite plug (pipette) open to air. The toluene was thereafter removed in vacuo ( $\approx 1 \mathrm{mmHg}$ ) to obtain pure product as a solid or oil. For the isomerization of 3-penten-2-ol, the above was carried out in toluene- $d_{8}$ and the pure product was characterized directly with NMR spectroscopy after passing the reaction mixture through the celite plug.

Characterization of products: description, (\% yield). Characterization; ${ }^{1} \mathrm{H}$ NMR spectrum (top), ${ }^{13 \mathrm{C}} \mathrm{NMR}$ spectrum (bottom). Residual NMR solvent peaks indicated with *.

1-phenylethan-1-ol (1aH $\mathbf{1 a H}_{2}$. Colorless, clear oil (22 mg, 99\% isolated yield). ${ }^{1} \mathrm{H} \mathrm{NMR}\left(300 \mathrm{MHz}, \mathrm{CDCl}_{3}\right)$ $\delta 7.44-7.23(\mathrm{~m}, 5 \mathrm{H}), 4.90(\mathrm{qd}, J=6.4,3.4 \mathrm{~Hz}, 1 \mathrm{H}), 1.92(\mathrm{~s}, 1 \mathrm{H}), 1.5 \mathrm{O}(\mathrm{d}, J=6.5 \mathrm{~Hz}, 3 \mathrm{H}){ }^{3} \mathrm{C}$ NMR $(75 \mathrm{MHz}$, $\left.\mathrm{CDCl}_{3}\right) \delta 145.8,128.5,127.5,125.4,70.4,25.2$.

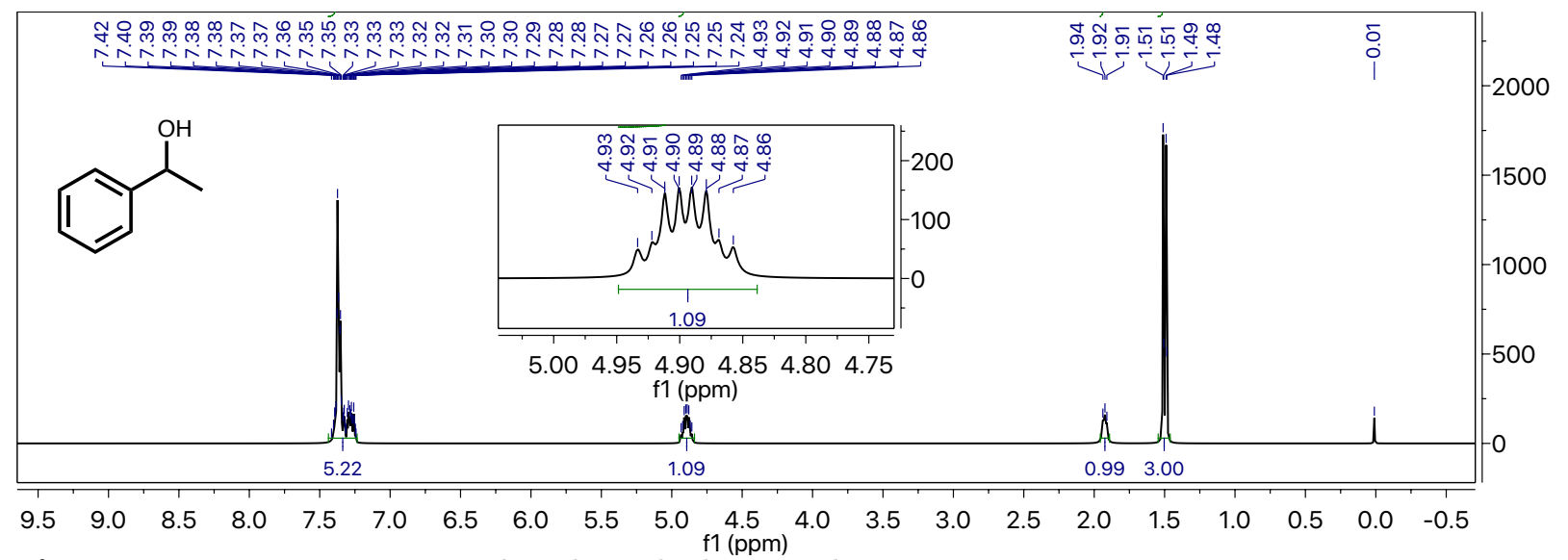

Figure S9. ${ }^{1} \mathrm{H}$ NMR spectrum of 1-phenylethan-1-ol. 


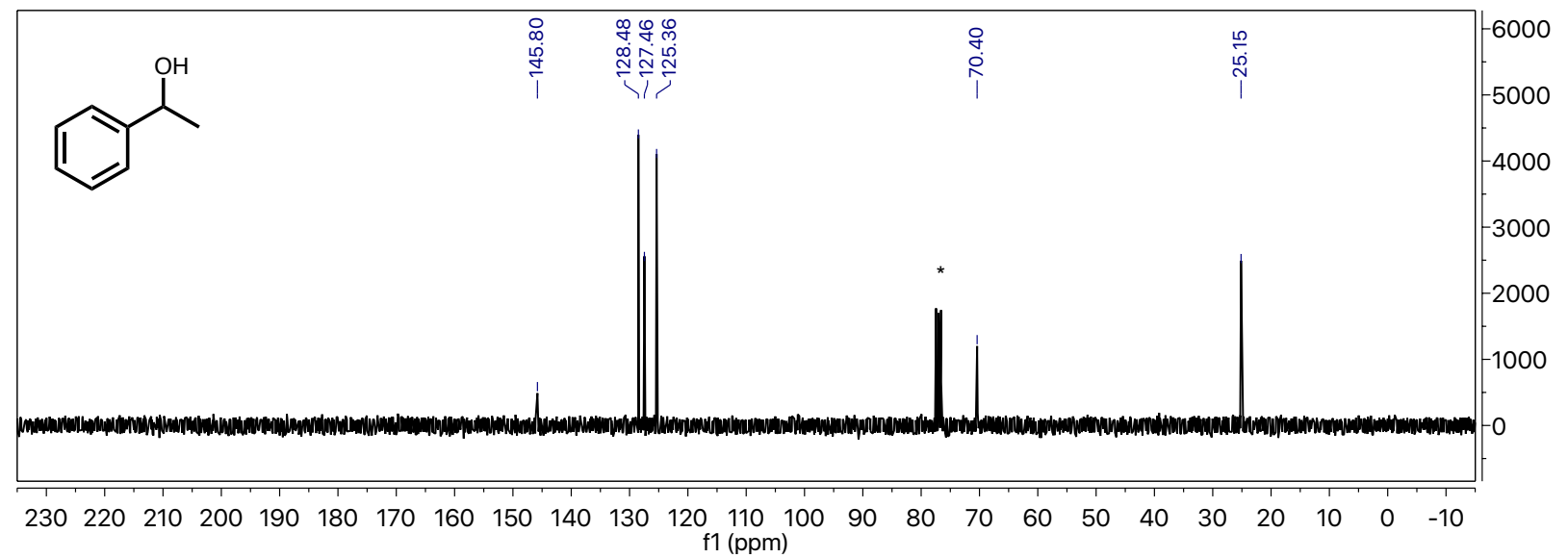

Figure S10. ${ }^{13} \mathrm{C}$ NMR spectrum of 1-phenylethan-1-ol.

1-(2-bromophenyl)ethan-1-ol (1bH2). Colorless, clear, viscous oil. (38 mg, 99\% isolated yield). ${ }^{1} \mathrm{H}$ $\operatorname{NMR}\left(300 \mathrm{MHz}, \mathrm{CDCl}_{3}\right) \delta 7.56(\mathrm{~d}, J=7.8 \mathrm{~Hz}, 1 \mathrm{H}), 7.49(\mathrm{~d}, J=8.0 \mathrm{~Hz}, 1 \mathrm{H}), 7.32(\mathrm{t}, J=7.6 \mathrm{~Hz}, 1 \mathrm{H}), 7.10(\mathrm{t}$, $J=7.7 \mathrm{~Hz}, 1 \mathrm{H}), 5.20(\mathrm{q}, J=6.4 \mathrm{~Hz}, 1 \mathrm{H}), 2.47(\mathrm{~s}, 1 \mathrm{H}), 1.45(\mathrm{~d}, J=6.4 \mathrm{~Hz}, 3 \mathrm{H}) .{ }^{13 \mathrm{C}} \mathrm{NMR}\left(75 \mathrm{MHz}, \mathrm{CDCl}_{3}\right) \delta$ $144.6,132.6,128.7,127.8,126.7,121.7,69.1,23.6$.

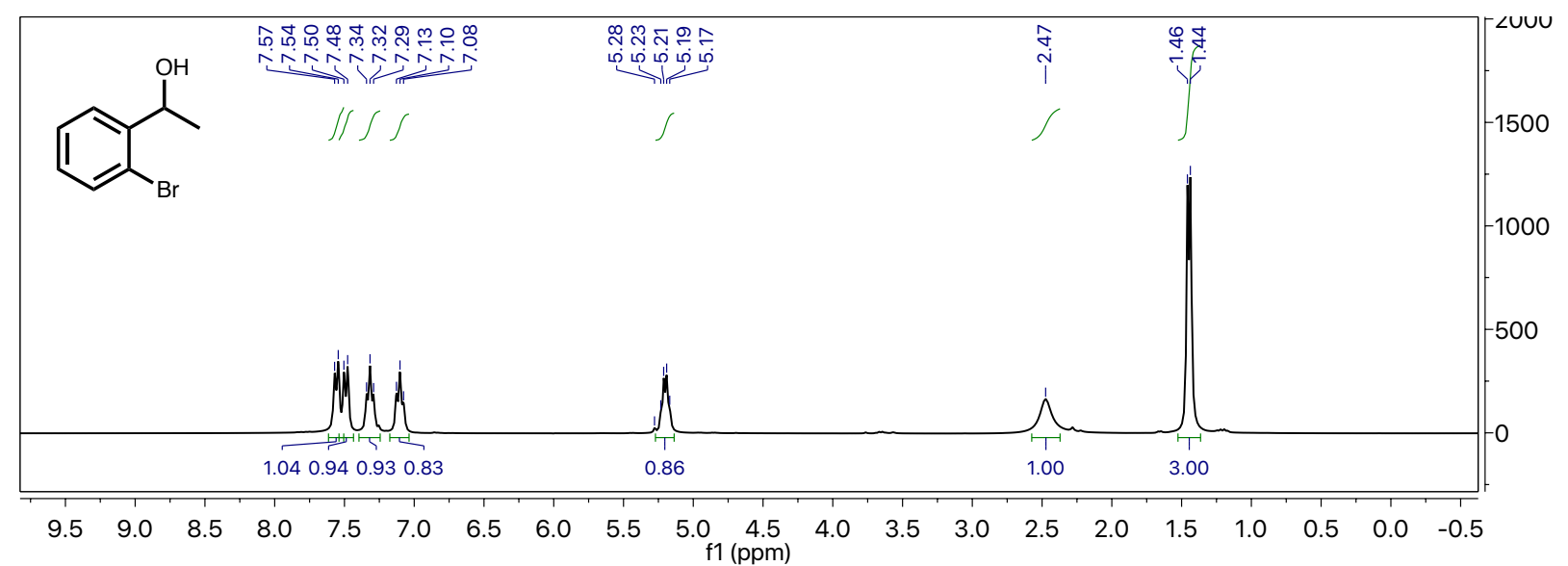

Figure S11. ${ }^{1} \mathrm{H}$ NMR spectrum of 1-(2-bromophenyl)ethan-1-ol.

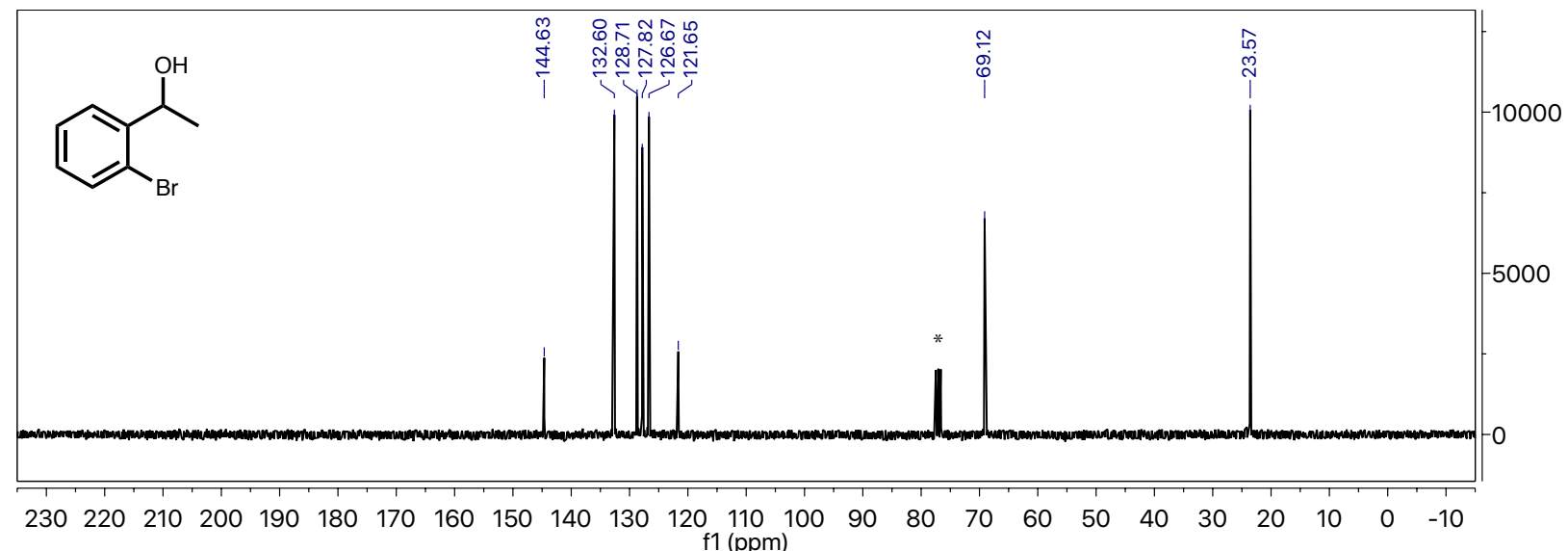

Figure S12. ${ }^{13} \mathrm{C}$ NMR spectrum of 1-(2-bromophenyl)ethan-1-ol.

1-(4-methoxyphenyl)ethan-1-ol (1cH2). Colorless, clear oil (25 mg, 84\% isolated yield). ${ }^{1} \mathrm{H}$ NMR (300 $\left.\mathrm{MHz}, \mathrm{CDCl}_{3}\right) \delta 7.28(\mathrm{~d}, J=8.4 \mathrm{~Hz}, 2 \mathrm{H}), 6.87(\mathrm{~d}, J=8.8,2 \mathrm{H}), 4.84(\mathrm{q}, J=6.5 \mathrm{~Hz}, 1 \mathrm{H}), 3.79(\mathrm{~s}, 3 \mathrm{H}), 1.47(\mathrm{~d}$, $J=6.4,3 \mathrm{H}) .{ }^{13 \mathrm{C}} \mathrm{NMR}\left(75 \mathrm{MHz}, \mathrm{CDCl}_{3}\right) \delta$ 158.94, 137.97, 126.64, 113.82, 69.96, 55.28, 24.99. 


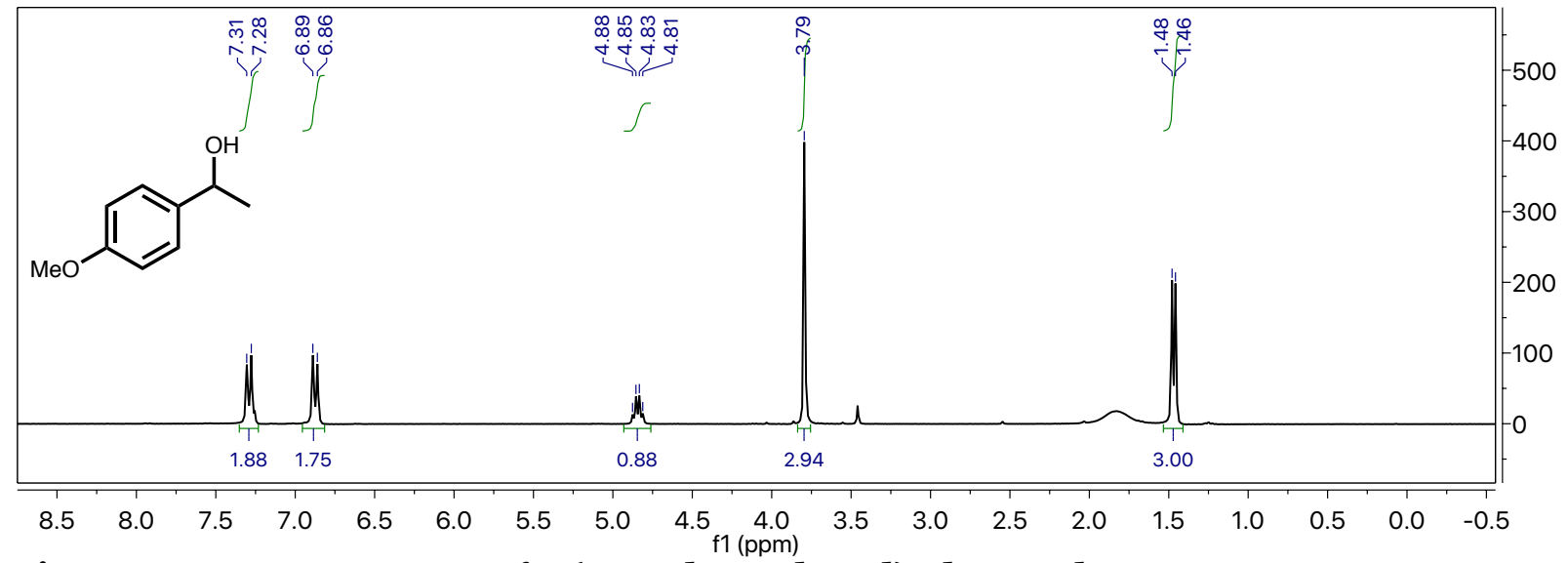

Figure S13. ${ }^{1 \mathrm{H}}$ NMR spectrum of 1-(4-methoxyphenyl)ethan-1-ol.

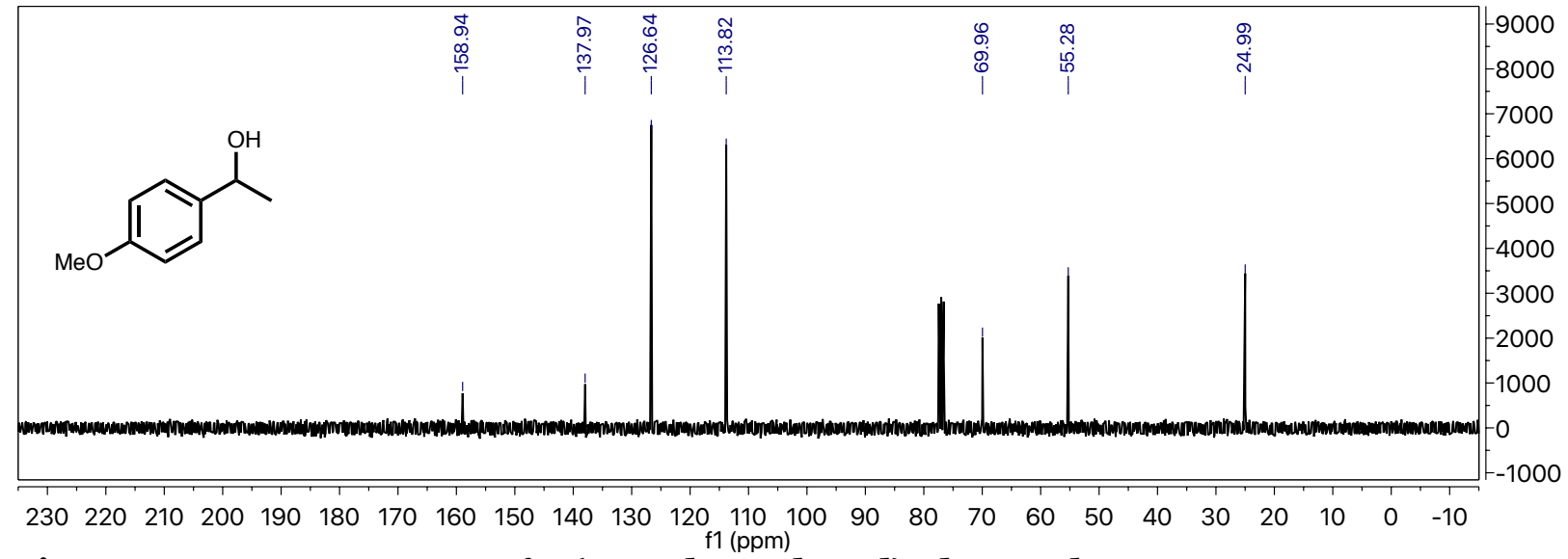

Figure S14. ${ }^{13 \mathrm{C}}$ NMR spectrum of 1-(4-methoxyphenyl)ethan-1-ol.

1-(4-nitrophenyl)ethan-1-ol (1dH2). Yellow oil (23 mg, 71\% isolated yield). ${ }^{1} \mathrm{H}$ NMR (30O MHz, $\left.\mathrm{CDCl}_{3}\right) \delta 8.17(\mathrm{~d}, J=8.8 \mathrm{~Hz}, 2 \mathrm{H}), 7.54(\mathrm{~d}, J=8.8 \mathrm{~Hz}, 2 \mathrm{H}), 5.00(\mathrm{qd}, J=6.5,3.2 \mathrm{~Hz}, 1 \mathrm{H}), 1.50(\mathrm{~d}, J=6.5$ $\mathrm{Hz}, 3 \mathrm{H}) .{ }^{13 \mathrm{C}} \mathrm{NMR}\left(75 \mathrm{MHz}, \mathrm{CDCl}_{3}\right.$ ) $\delta$ 153.12, 126.10, 123.71, 69.45, 25.47.

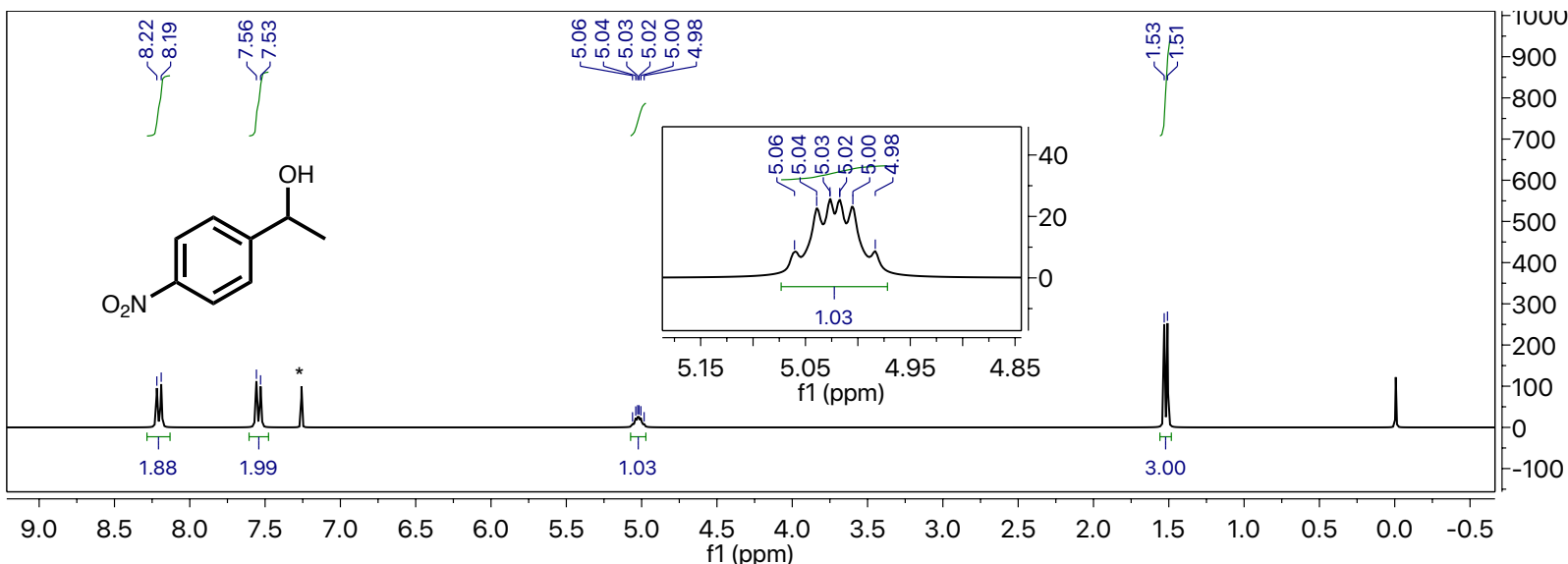

Figure S15. ${ }^{1} \mathrm{H}$ NMR spectrum of 1-(4-nitrophenyl)ethan-1-ol. 


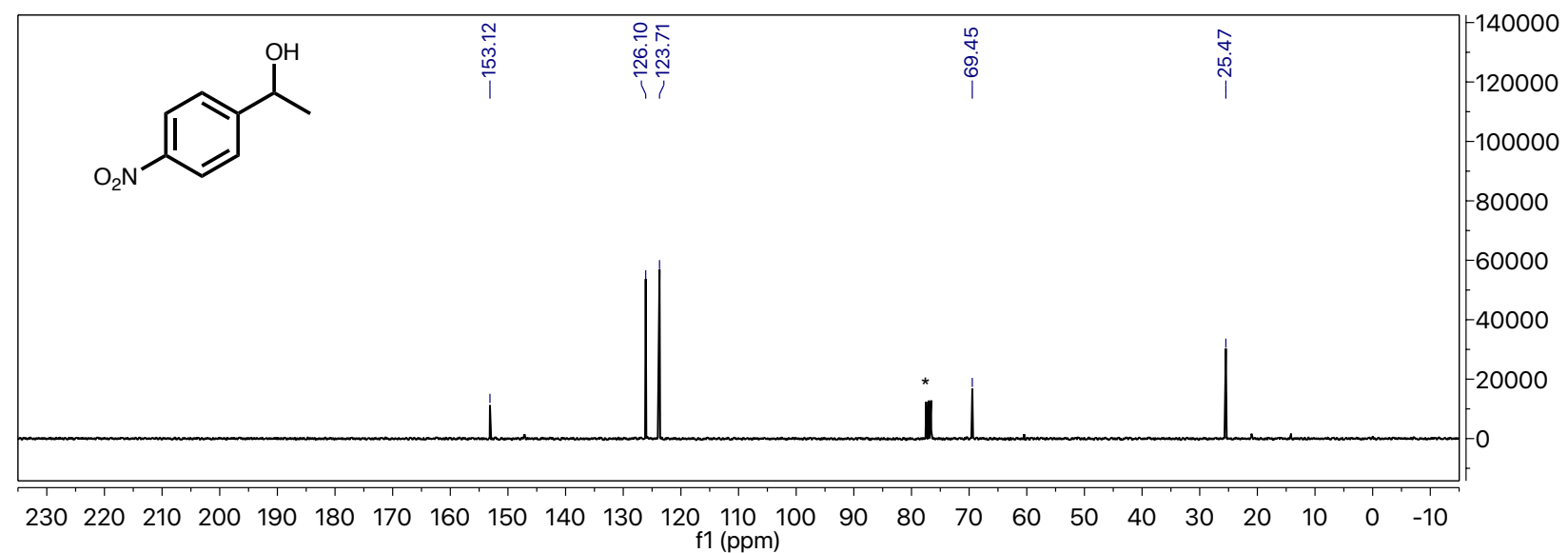

Figure S16. ${ }^{13 \mathrm{C}}$ NMR spectrum of 1-(4-nitrophenyl)ethan-1-ol.

1-(4-methylphenyl)ethan-1-ol (1eH2). Catalysis performed at higher scale (o.38 mmol ketone). Light yellow, clear, light oil (51 mg, 99\% isolated yield). ${ }^{1} \mathrm{H} \mathrm{NMR}\left(300 \mathrm{MHz}, \mathrm{CDCl}_{3}\right) \delta 7.25$ (d, $\left.J=7.8 \mathrm{~Hz}, 2 \mathrm{H}\right)$, $7.15(\mathrm{~d}, J=7.9 \mathrm{~Hz}, 2 \mathrm{H}), 4.83(\mathrm{q}, J=6.5 \mathrm{~Hz}, 1 \mathrm{H}), 2.34(\mathrm{~s}, 3 \mathrm{H}), 1.93(\mathrm{~s}, 1 \mathrm{H}), 1.47(\mathrm{~d}, J=6.5 \mathrm{~Hz}, 3 \mathrm{H}) .13 \mathrm{C} \mathrm{NMR}$ $\left(75 \mathrm{MHz}, \mathrm{CDCl}_{3}\right) \delta 142.88,137.10,129.13,125.34,70.21,25.08,21.09$.

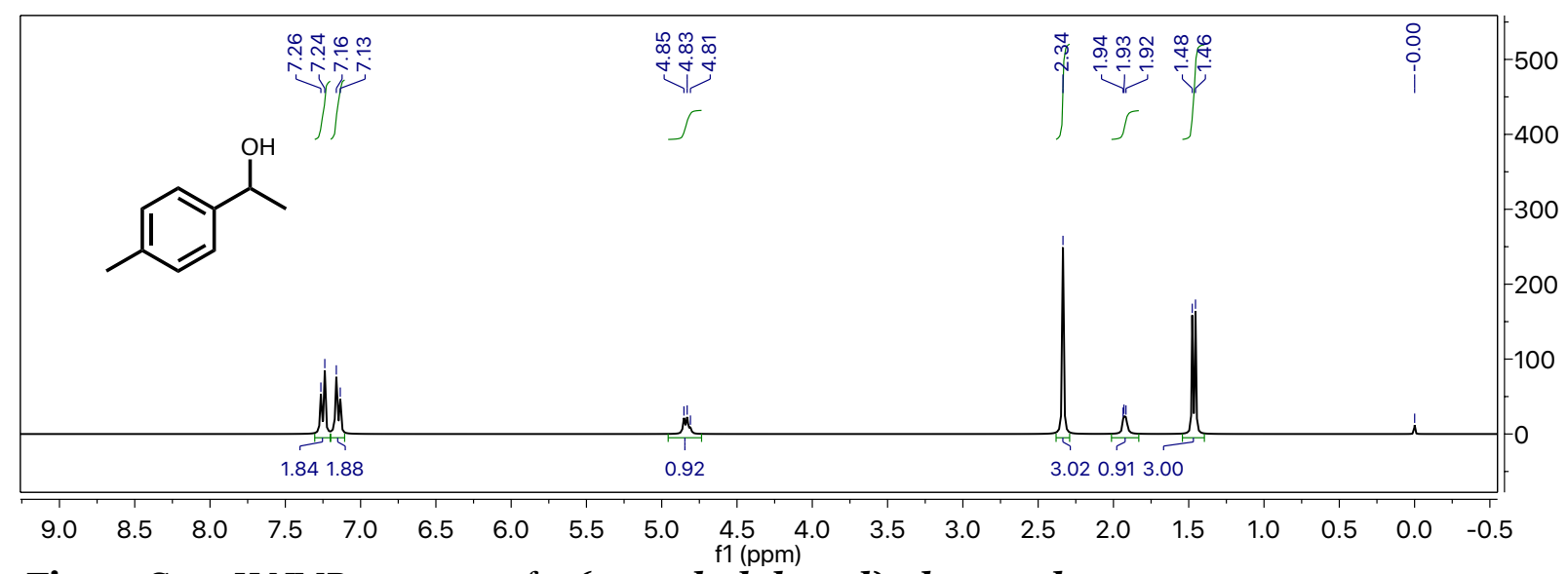

Figure S17. ${ }^{1} \mathrm{H}$ NMR spectrum of 1-(4-methylphenyl)ethan-1-ol.

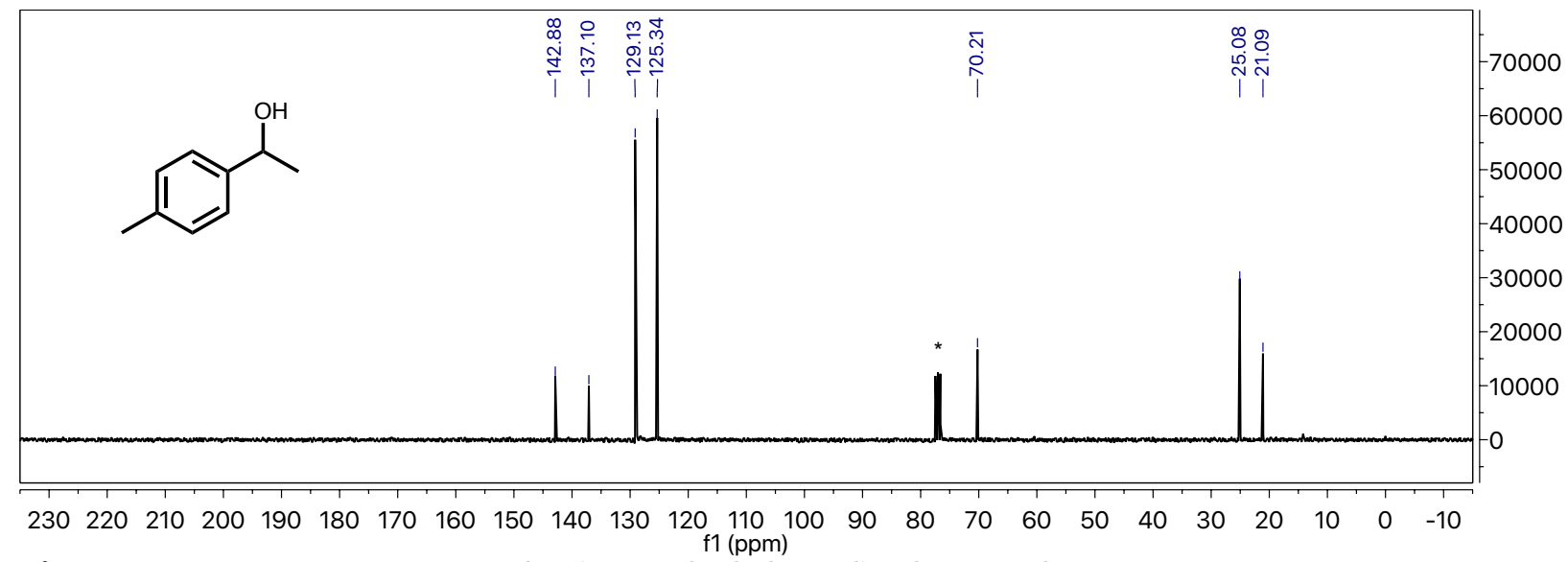

Figure S18. ${ }^{13 \mathrm{C}}$ NMR spectrum of 1-(4-methylphenyl)ethan-1-ol.

1-(4-(allyloxy)-phenyl)ethan-1-ol (1fH2). Colorless, clear oil (66 mg, 98\% isolated yield). ${ }^{1} \mathrm{H}$ NMR $\left(400 \mathrm{MHz}, \mathrm{CDCl}_{3}\right) \delta 7.28(\mathrm{~d}, J=8.6 \mathrm{~Hz}, 4 \mathrm{H}), 6.89(\mathrm{~d}, J=8.4 \mathrm{~Hz}, 2 \mathrm{H}), 6.04$ (ddd, $J=16.8,11.6,6.3 \mathrm{~Hz}$, $1 \mathrm{H}), 5.41(\mathrm{~d}, J=17.3 \mathrm{~Hz}, 1 \mathrm{H}), 5.28(\mathrm{~d}, J=10.4 \mathrm{~Hz}, 1 \mathrm{H}), 4.85(\mathrm{p}, J=5.9 \mathrm{~Hz}, 1 \mathrm{H}), 4.53(\mathrm{~d}, J=4.1 \mathrm{~Hz}, 3 \mathrm{H})$, 
$1.46(\mathrm{~d}, J=6.4 \mathrm{~Hz}, 3 \mathrm{H}) .{ }^{13 \mathrm{C}} \mathrm{NMR}\left(101 \mathrm{MHz}, \mathrm{CDCl}_{3}\right) \delta 138.11,133.26,126.62,117.63,114.67,109.99,69.97$, $68.84,24.97$.

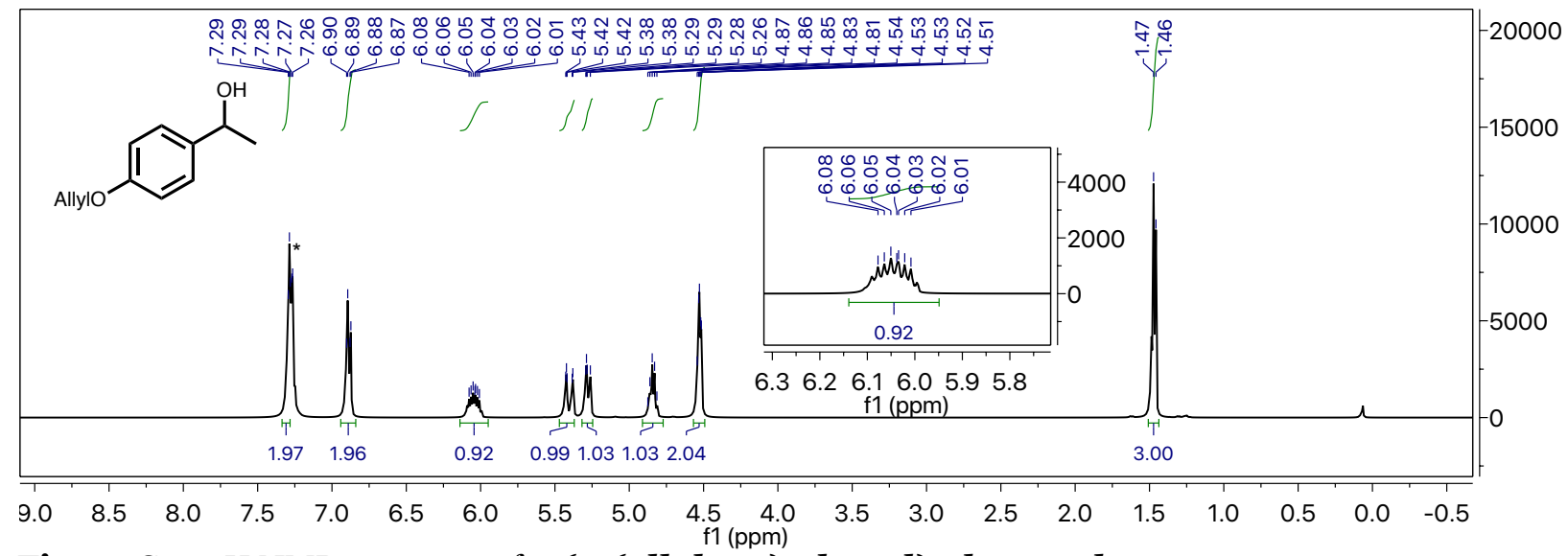

Figure S19. ${ }^{1} \mathrm{H}$ NMR spectrum of 1-(4-(allyloxy)-phenyl)ethan-1-ol.

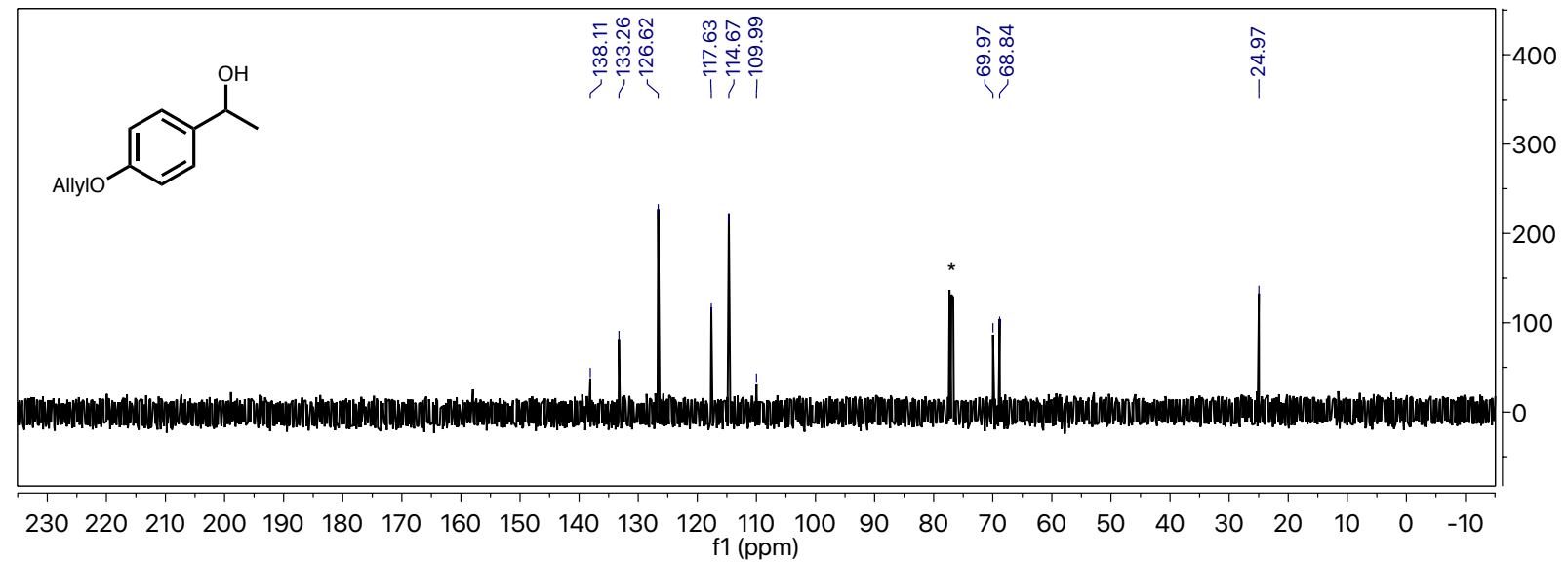

Figure S20. ${ }^{13} \mathrm{C}$ NMR spectrum of 1-(4-(allyloxy)-phenyl)ethan-1-ol.

1-(3-(allyloxy)-phenyl)ethan-1-ol (1gH2). Catalysis was performed at higher scale (1.53 mmol ketone). Brown, viscous oil (244 mg, 94\% isolated yield). $\left.{ }^{1} \mathrm{H} \mathrm{NMR} \mathrm{(300} \mathrm{MHz,} \mathrm{CDCl}_{3}\right) \delta 7.25$ (t, $J=7.2 \mathrm{~Hz}$, $1 \mathrm{H}), 6.97-6.92(\mathrm{~m}, 2 \mathrm{H}), 6.82(\mathrm{~d}, J=8.5 \mathrm{~Hz}, 1 \mathrm{H}), 6.06(\mathrm{tdd}, J=16.1,11.2,6.2 \mathrm{~Hz}, 1 \mathrm{H}), 5.42(\mathrm{~d}, J=17.3 \mathrm{~Hz}$, $1 \mathrm{H}), 5.28(\mathrm{~d}, J=10.5 \mathrm{~Hz}, 1 \mathrm{H}), 4.87(\mathrm{q}, J=6.4 \mathrm{~Hz}, 1 \mathrm{H}), 4.55(\mathrm{~d}, J=5.1 \mathrm{~Hz}, 2 \mathrm{H}), 1.83(\mathrm{~s}, 1 \mathrm{H}), 1.48(\mathrm{~d}, J=6.7$ $\mathrm{Hz}, 3 \mathrm{H}) .{ }^{13 \mathrm{C}} \mathrm{NMR}\left(75 \mathrm{MHz}, \mathrm{CDCl}_{3}\right.$ ) $\delta 158.78,147.54,133.24,129.50,117.82,117.65,113.61,111.77,70.32$, $68.75,25.11$.

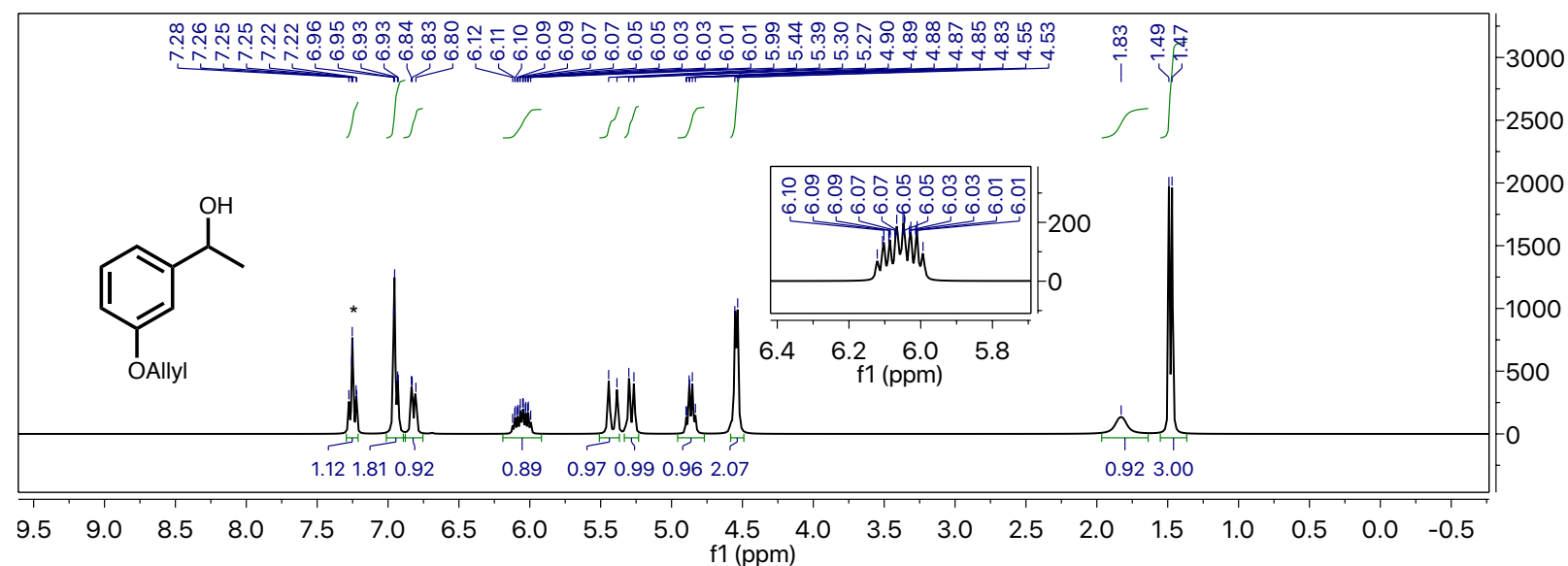

Figure S21. ${ }^{1} \mathrm{H}$ NMR spectrum of 1-(3-(allyloxy)-phenyl)ethan-1-ol. 


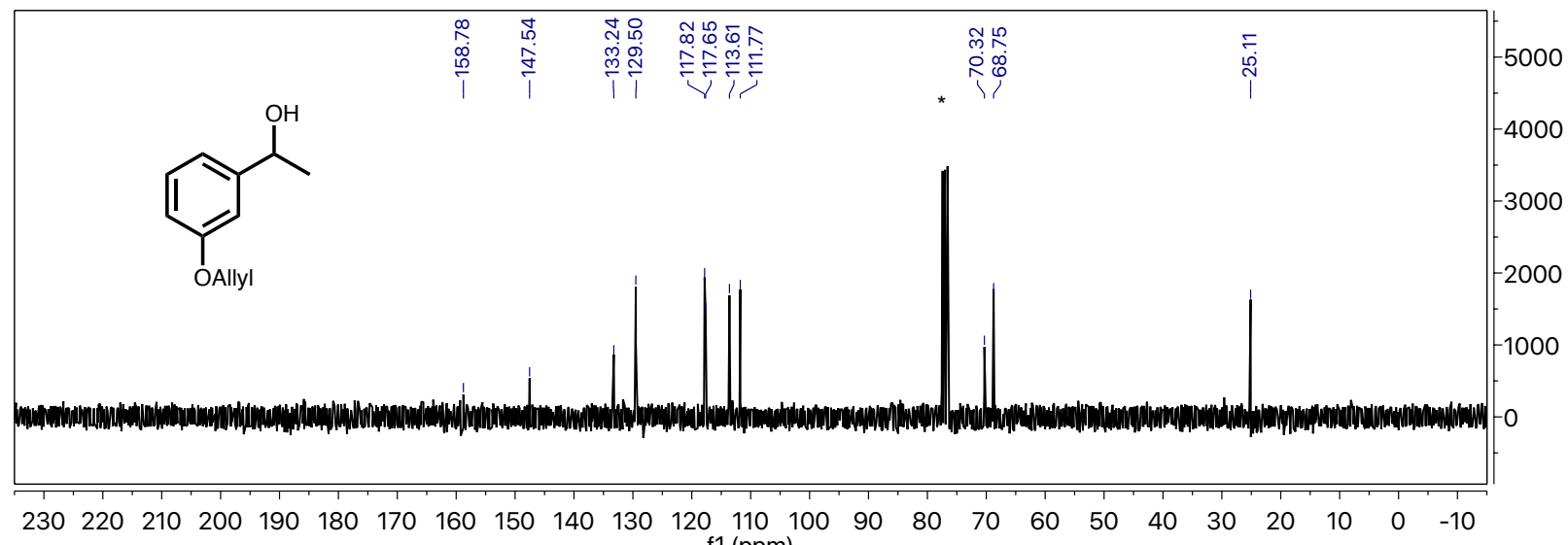

Figure S22. ${ }^{13}$ C NMR spectrum of 1-(3-(allyloxy)-phenyl)ethan-1-ol.

1,2-diphenylethan-1-ol (1iH2). White solid. (33 mg, 87\% isolated yield). ${ }^{1} \mathrm{H} \mathrm{NMR}\left(300 \mathrm{MHz}, \mathrm{CDCl}_{3}\right) \delta$ $=7.37-7.19(\mathrm{~m}, 1 \mathrm{OH}, \mathrm{Ar}-H), 4.91(\mathrm{q}, 1 \mathrm{H}, \mathrm{C}-H), 2.99(\mathrm{~m}, 2 \mathrm{H}, \mathrm{C}-H), 1.95(\mathrm{sh} \mathrm{s}, 1 \mathrm{H}, \mathrm{O}-\mathrm{H}) .{ }^{13 \mathrm{C}} \mathrm{NMR}(75 \mathrm{MHz}$, $\left.\mathrm{CDCl}_{3}\right) \delta=143.8,138.0,129.5,128.5,128.4,127.6,126.6,125.9,75.3,46.1$. (+ denotes residual ethyl acetate).

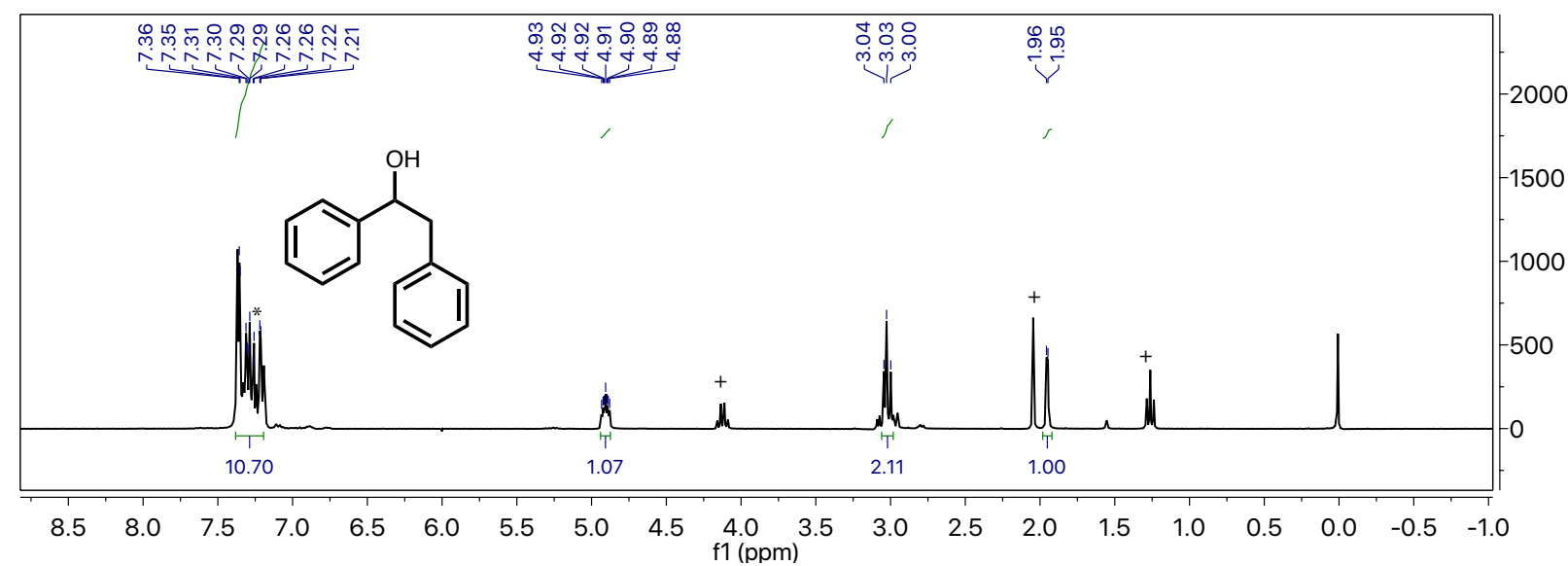

Figure S23. ${ }^{1 \mathrm{H}}$ NMR spectrum of 1,2-diphenylethan-1-ol.

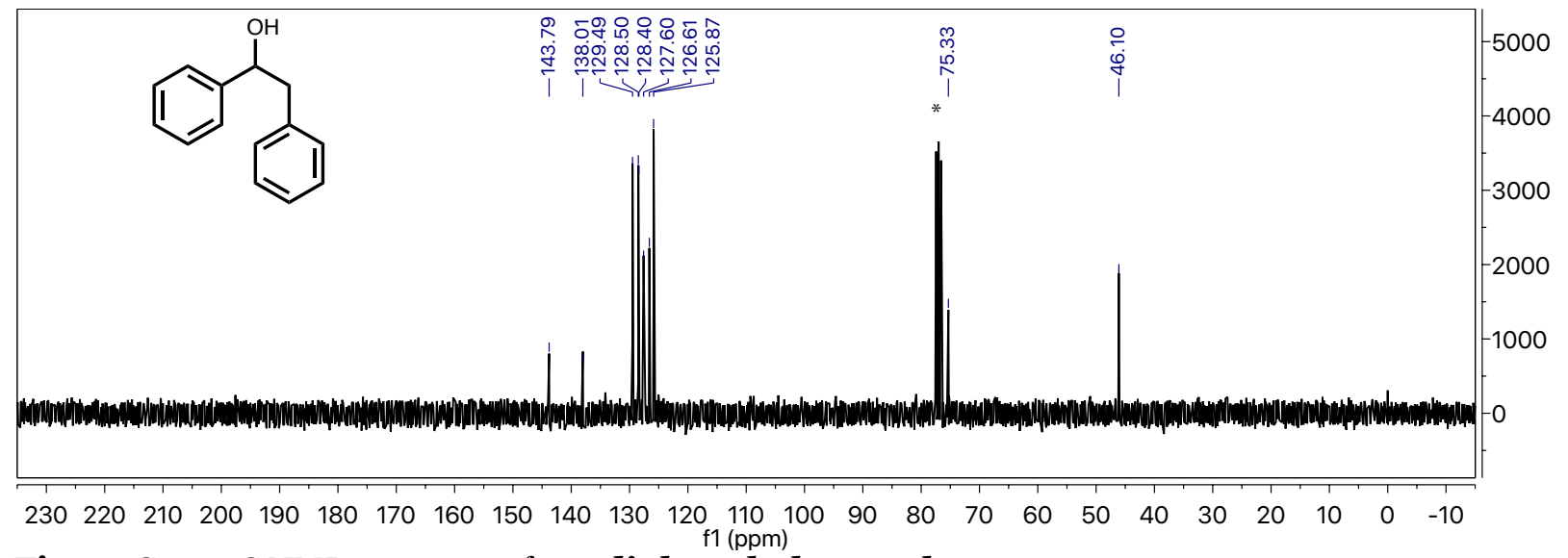

Figure S24. ${ }^{13} \mathrm{C}$ NMR spectrum of 1,2-diphenylethan-1-ol.

(4-bromophenyl)(phenyl)methanol (2aH2). Off-white solid (41 mg, 84\% isolated yield). ${ }^{1 \mathrm{H}} \mathrm{NMR}$ $\left(300 \mathrm{MHz}, \mathrm{CDCl}_{3}\right) \delta 7.46(\mathrm{~d}, J=8.5 \mathrm{~Hz}, 2 \mathrm{H}), 7.35-7.27(\mathrm{~m}, 5 \mathrm{H}), 7.26(\mathrm{~d}, J=8.2 \mathrm{~Hz}, 2 \mathrm{H}), 5.79(\mathrm{~d}, J=3.5$ $\mathrm{Hz}, 2 \mathrm{H}), 2.26$ (s, $1 \mathrm{H}) .{ }^{13 \mathrm{C} \mathrm{NMR}}\left(75 \mathrm{MHz}, \mathrm{CDCl}_{3}\right) \delta=143.3,142.7,131.5,128.6,128.2,127.9,126.5 .121 .4$, 75.6 . 


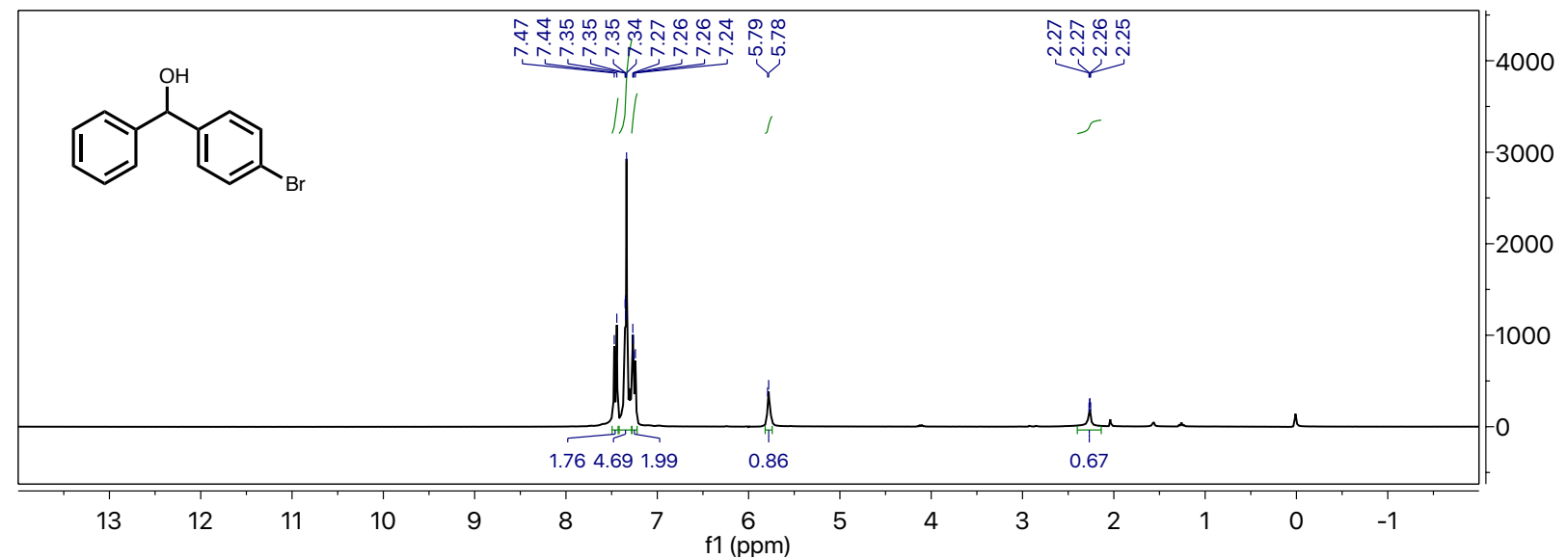

Figure S25. ${ }^{1 \mathrm{H}}$ NMR spectrum of (4-bromophenyl)(phenyl)methanol.

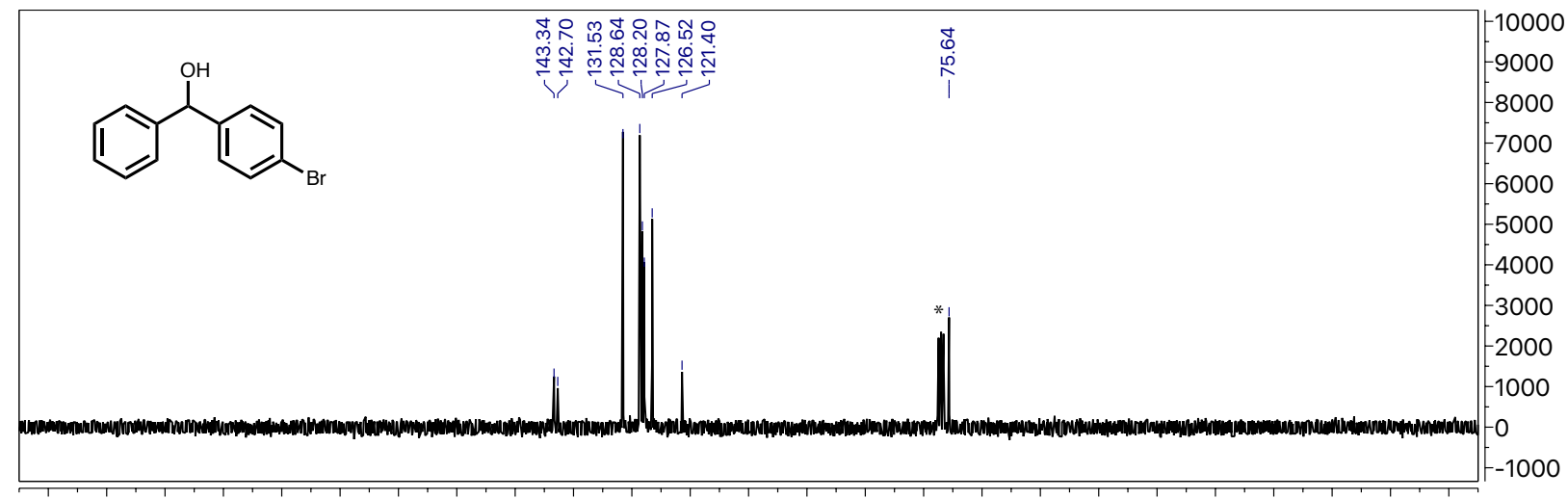

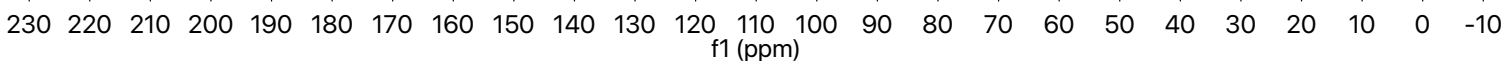

Figure S26. ${ }^{13 \mathrm{C}}$ NMR spectrum of (4-bromophenyl)(phenyl)methanol.

Diphenylmethanol (2bH2). White solid (182 mg, 99\% isolated yield). ${ }^{1} \mathrm{H}$ NMR (300 $\left.\mathrm{MHz}, \mathrm{CDCl}_{3}\right) \delta$ 7.40-7.23 (m, 10H), $5.85(\mathrm{~d}, J=3.4 \mathrm{~Hz}, 1 \mathrm{H}), 2.22(\mathrm{~d}, J=3.5 \mathrm{~Hz}, 1 \mathrm{H}) .{ }^{13} \mathrm{C}$ NMR $\left(75 \mathrm{MHz}, \mathrm{CDCl}_{3}\right) \delta 143.8$, $128.5,127.6,126.5,76.3$.

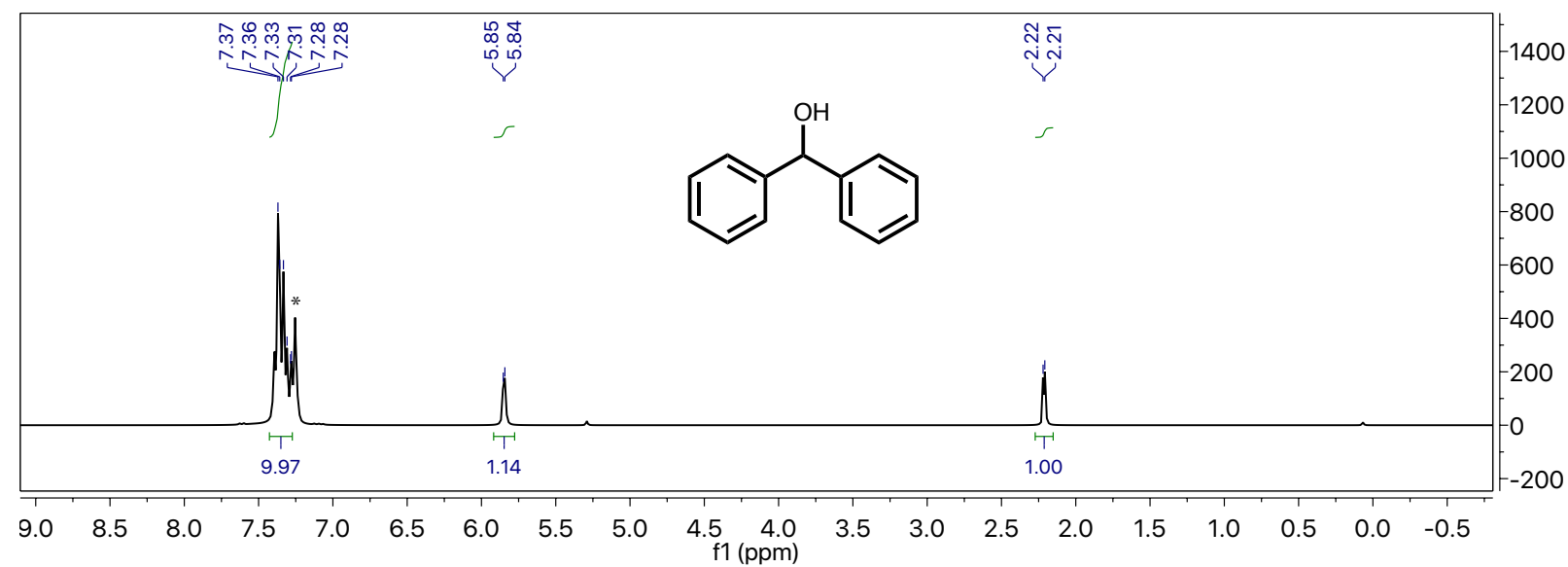

Figure S27. ${ }^{1 \mathrm{H}}$ NMR spectrum of diphenylmethanol. 


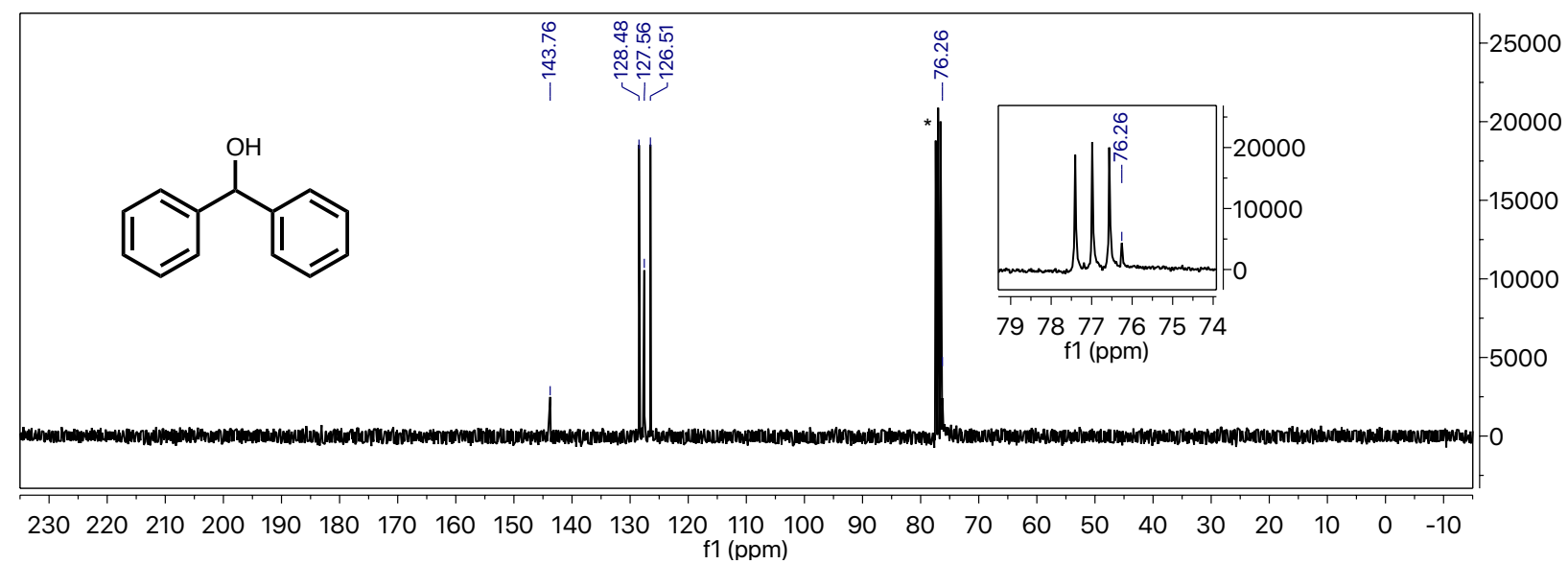

Figure S28. ${ }^{13} \mathrm{C}$ NMR spectrum of diphenylmethanol.

2-octanol (3aH2). Catalysis was performed at higher scale (0.95 mmol ketone) Colorless, clear oil (111 $\mathrm{mg}, 90 \%$ isolated yield, isolated through silica plug with hexane). ${ }^{1} \mathrm{H} \mathrm{NMR}\left(300 \mathrm{MHz}, \mathrm{CDCl}_{3}\right) \delta=3.77$ (q, $1 \mathrm{H}, \mathrm{C}-\mathrm{H}), 1.41-1.25\left(\mathrm{~m}, 12 \mathrm{H}, \mathrm{C}-\mathrm{H}_{2}\right), 1.17\left(\mathrm{~d}, 3 \mathrm{H}, \mathrm{C}-\mathrm{H}_{3}\right), 0.85\left(\mathrm{~d}, 3 \mathrm{H}, \mathrm{C}-\mathrm{H}_{3}\right) .{ }^{13} \mathrm{C} \mathrm{NMR}\left(75 \mathrm{MHz}, \mathrm{CDCl}_{3}\right) \delta=$ 68.1, 39.4, 31.8, 29.3, 25.1, 23.4, 22.6, 14.0.

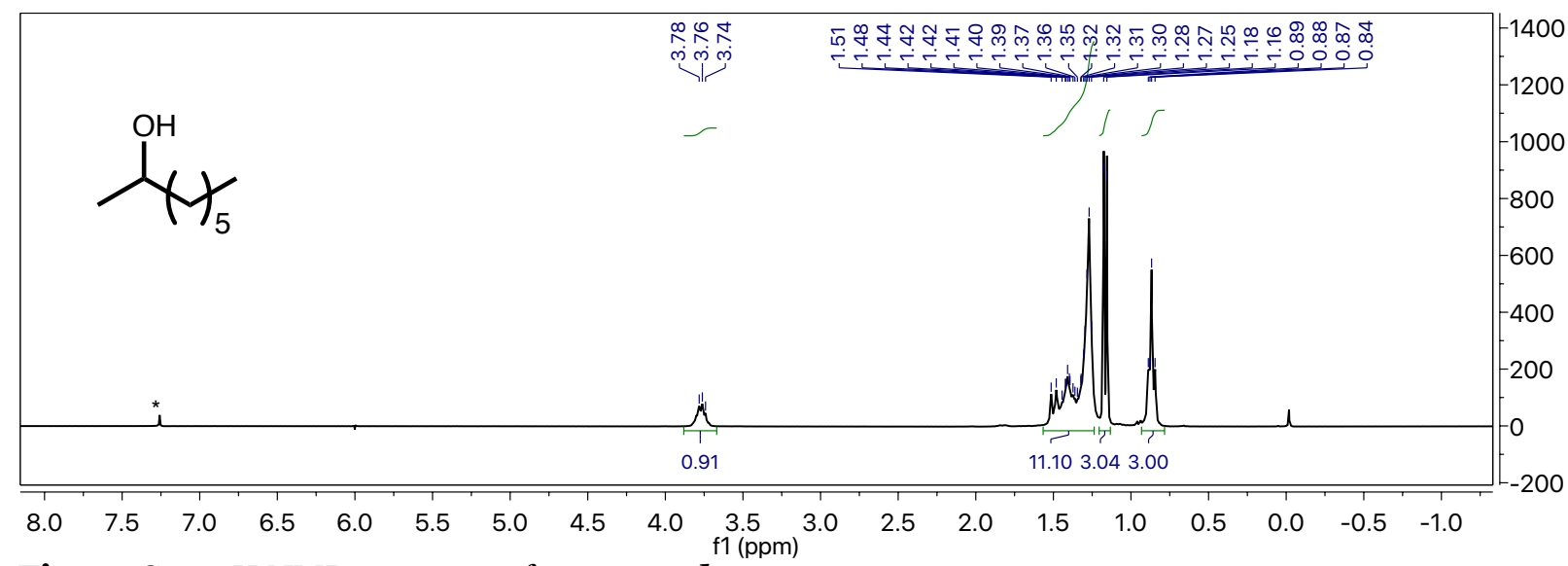

Figure S29. ${ }^{1} \mathrm{H}$ NMR spectrum of 2-octanol.

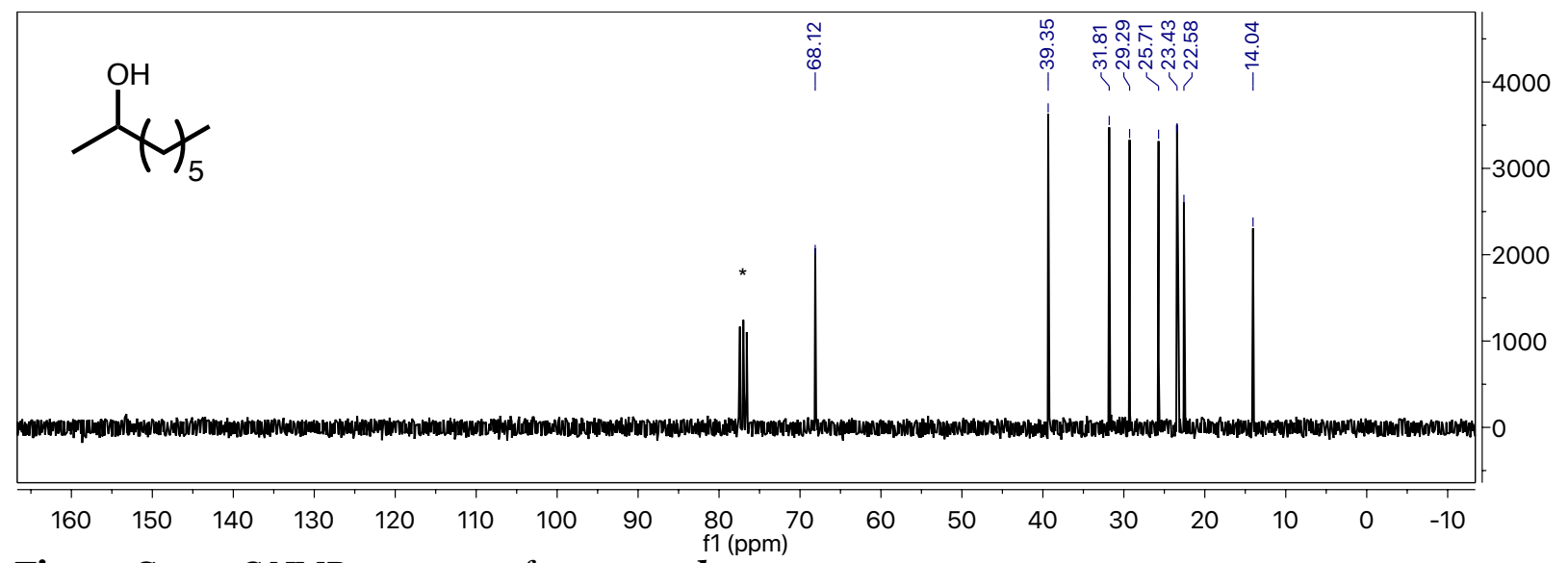

Figure S3o. ${ }^{13 \mathrm{C}}$ NMR spectrum of 2-octanol.

Cyclohexanol (3bH2). Colorless semi-solid - solidifies on standing. (193 $\mathrm{mg}$, 99\% isolated yield). ${ }^{1 \mathrm{H}}$ NMR (300 MHz, $\left.\mathrm{CDCl}_{3}\right) \delta 3.60(\mathrm{dp}, J=8.8,4.2 \mathrm{~Hz}, 1 \mathrm{H}), 2.03-1.83(\mathrm{~m}, 2 \mathrm{H}), 1.83-1.63(\mathrm{~m}, 2 \mathrm{H}), 1.57-$ $1.48(\mathrm{~m}, 3 \mathrm{H}), 1.35-1.16(\mathrm{~m}, 5 \mathrm{H}) .{ }^{13} \mathrm{C} \mathrm{NMR}\left(75 \mathrm{MHz}, \mathrm{CDCl}_{3}\right) \delta$ 70.33, 35.53, 25.43, 24.11. 


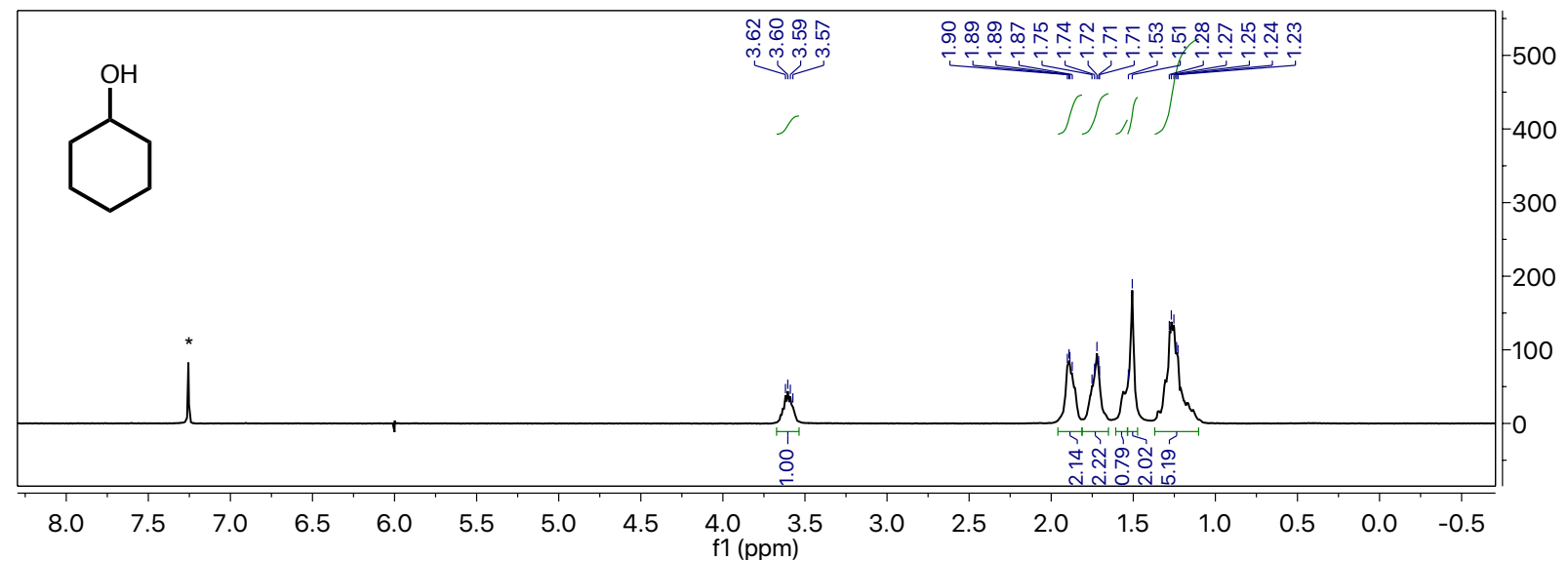

Figure S31. ${ }^{1} \mathrm{H}$ NMR spectrum of cyclohexanol.

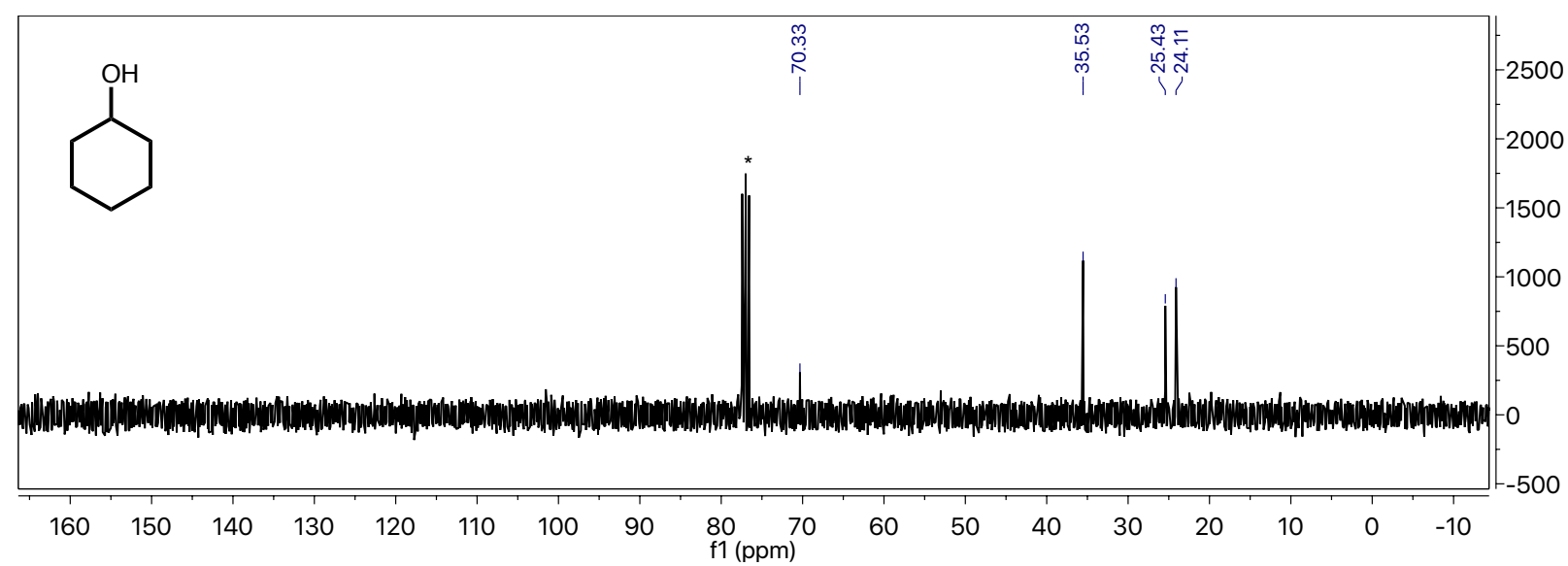

Figure S32. ${ }^{13 \mathrm{C}}$ NMR spectrum of cyclohexanol.

1-(thiophen-2-yl)ethan-1-ol (4aH2). Colorless, clear oil (19 mg, 85\% isolated yield). ${ }^{1} \mathrm{H}$ NMR (300 $\left.\mathrm{MHz}, \mathrm{CDCl}_{3}\right) \delta 7.34-7.13(\mathrm{~m}, 1 \mathrm{H}), 7.07-6.90(\mathrm{~m}, 2 \mathrm{H}), 5.13(\mathrm{q}, J=6.4 \mathrm{~Hz}, 1 \mathrm{H}), 2.00(\mathrm{~s}, 1 \mathrm{H}), 1.60(\mathrm{~d}, J=$ $6.4 \mathrm{~Hz}, 3 \mathrm{H}) .{ }^{13 \mathrm{C}} \mathrm{NMR}\left(75 \mathrm{MHz}, \mathrm{CDCl}_{3}\right) \delta 149.8,126.6,124.4,123.2,66.3,25.3$.

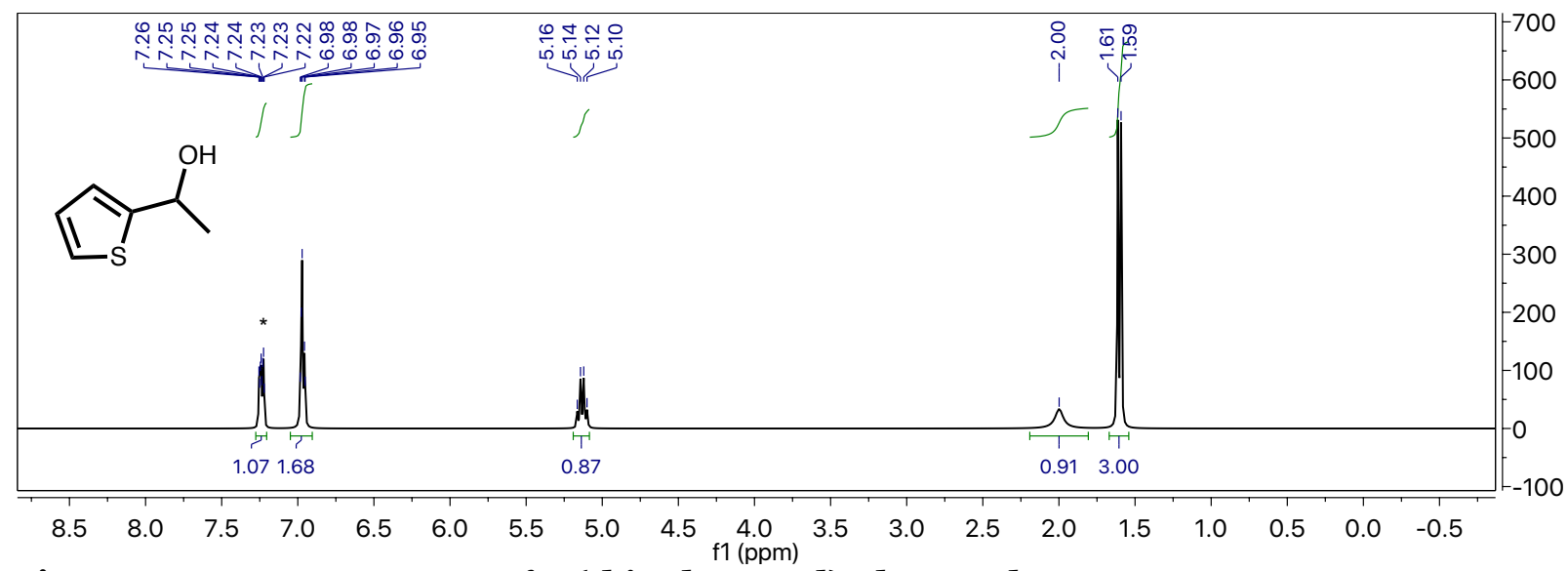

Figure S33. ${ }^{1} \mathrm{H}$ NMR spectrum of 1-(thiophen-2-yl)ethan-1-ol. 


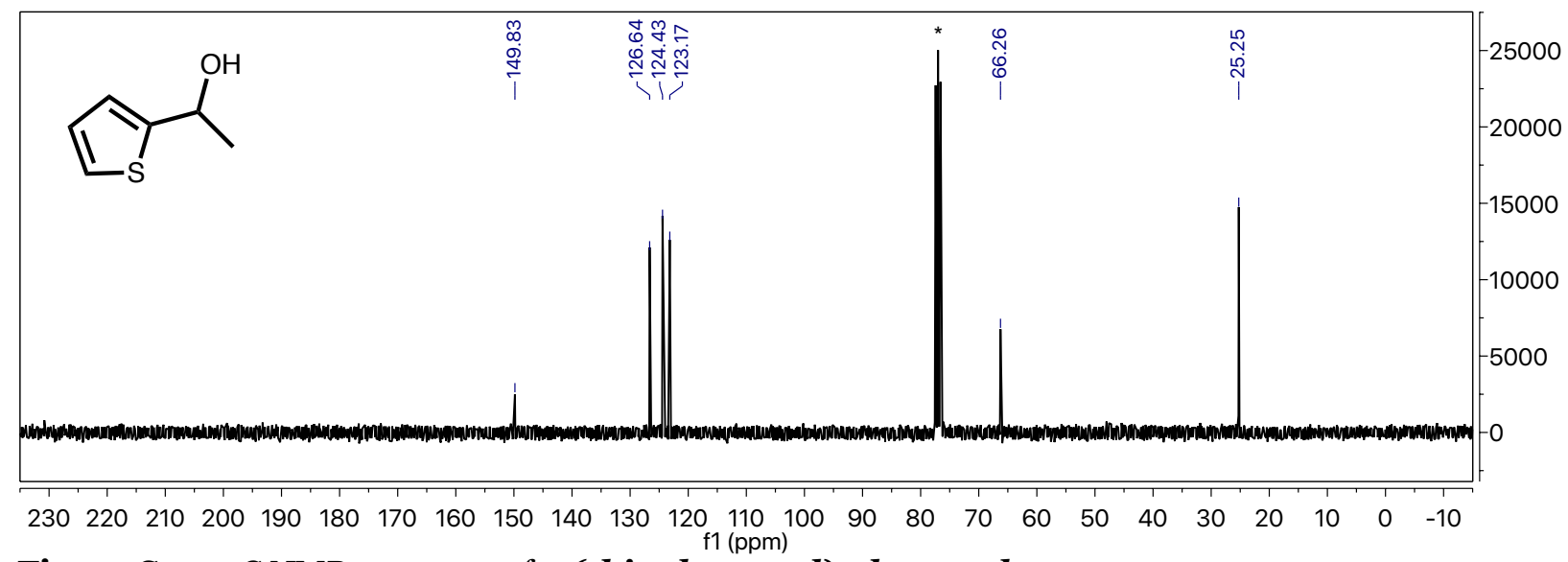

Figure S34. ${ }^{13 \mathrm{C}}$ NMR spectrum of 1-(thiophen-2-yl)ethan-1-ol.

1-(furan-2-yl)ethan-1-ol (4bH2). Catalysis was performed at a higher scale (Ketone - 0.38 mmol). Colorless, clear, light oil (26 mg, 63\% isolated yield). $\left.{ }^{1} \mathrm{H} \mathrm{NMR} \mathrm{(300} \mathrm{MHz,} \mathrm{CDCl}_{3}\right) \delta=7.36(\mathrm{~d}, 1 \mathrm{H}, \mathrm{Ar}-H)$, $6.32(\mathrm{~d}, 1 \mathrm{H}, \mathrm{Ar}-H), 6.21(\mathrm{dd}, 1 \mathrm{H}, \mathrm{Ar}-H), 4.87(\mathrm{q}, 1 \mathrm{H}, \mathrm{C}-\mathrm{H}), 2.08(\mathrm{br} \mathrm{s}, 1 \mathrm{H}, \mathrm{O}-\mathrm{H}), 1.53\left(\mathrm{~d}, 3 \mathrm{H}, \mathrm{C}-\mathrm{H}_{3}\right) .{ }_{13} \mathrm{C} \mathrm{NMR}$ $\left(75 \mathrm{MHz}, \mathrm{CDCl}_{3}\right) \delta=157.6,141.2,110.1,105.0,63.5,21.2$. (Peaks denoted by + from residual ethyl acetate).

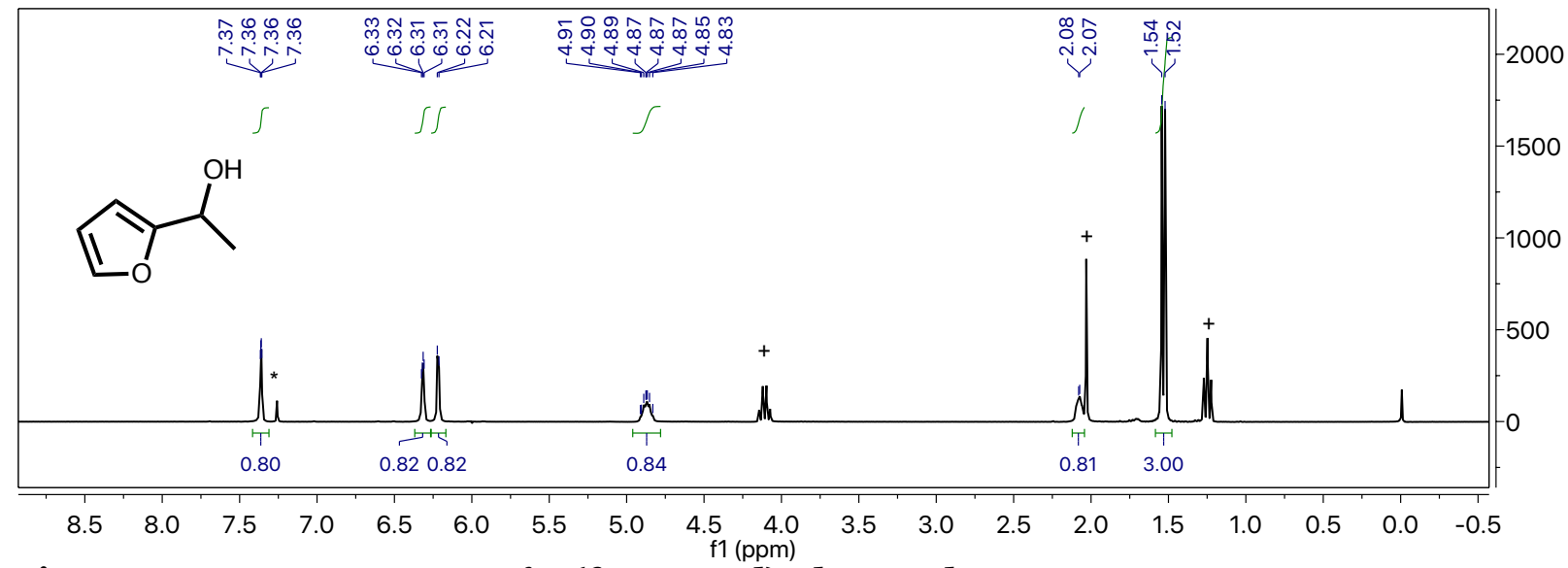

Figure S35. ${ }^{1} \mathrm{H}$ NMR spectrum of 1-(furan-2-yl)ethan-1-ol.

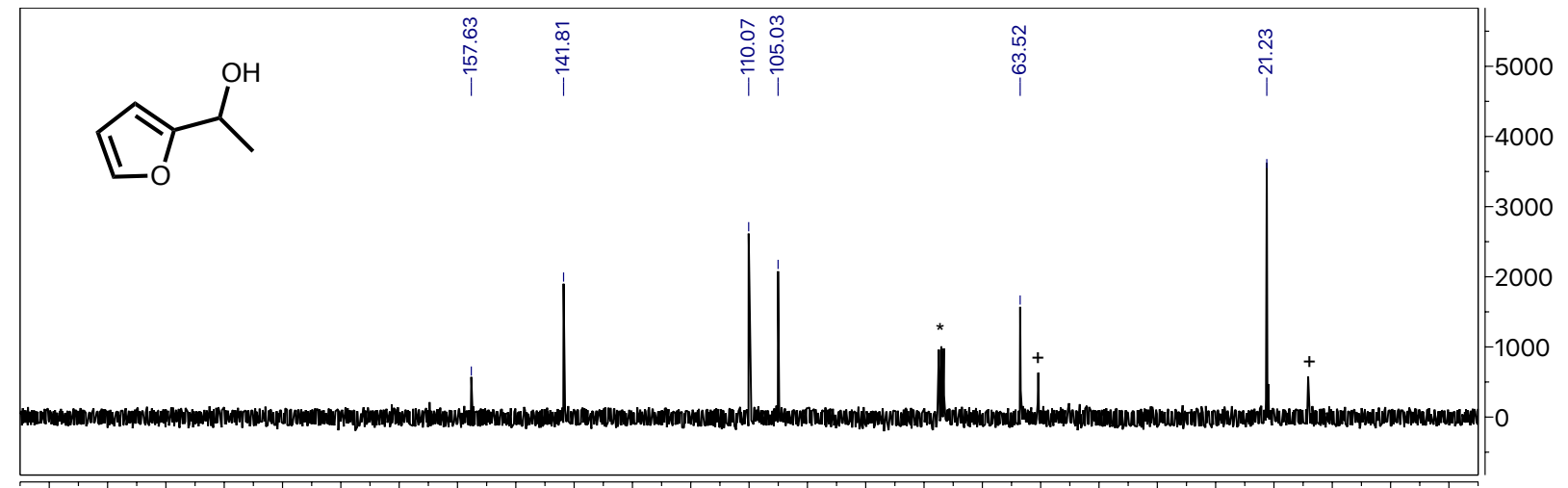

$\begin{array}{lllllllllllllllllllllllll}230 & 220 & 210 & 200 & 190 & 180 & 170 & 160 & 150 & 140 & 130 & 120 & 110 & 100 & 90 & 80 & 70 & 60 & 50 & 40 & 30 & 20 & 10 & 0 & -10\end{array}$

Figure S36. ${ }^{13} \mathrm{C}$ NMR spectrum of 1-(furan-2-yl)ethan-1-ol.

1-(thiophen-3-yl)ethan-1-ol (4cH2). Colorless, clear oil (22 mg, 89\% isolated yield). ${ }^{1} \mathrm{H}$ NMR (300 $\left.\mathrm{MHz}, \mathrm{CDCl}_{3}\right) \delta 7.30(\mathrm{dt}, J=5.5,2.8 \mathrm{~Hz}, 1 \mathrm{H}), 7.19(\mathrm{~d}, J=3.1 \mathrm{~Hz}, 1 \mathrm{H}), 7.12-7.07(\mathrm{~m}, 1 \mathrm{H}), 4.96(\mathrm{q}, J=6.5$ $\mathrm{Hz}, 1 \mathrm{H}), 1.52$ (d, $J=6.4 \mathrm{~Hz}, 3 \mathrm{H}) .{ }^{13 \mathrm{C}} \mathrm{NMR}\left(75 \mathrm{MHz}, \mathrm{CDCl}_{3}\right) \delta$ 147.27, 126.15, 125.59, 120.15, 66.55, 24.45. 


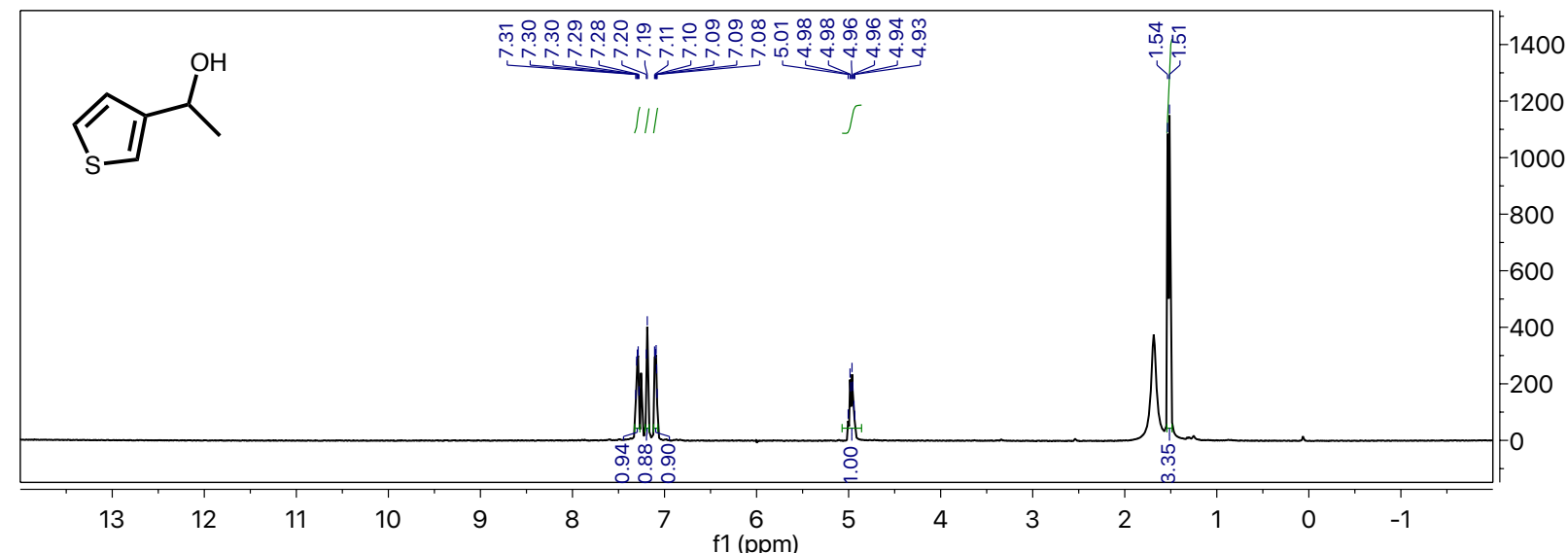

Figure S37. ${ }^{1} \mathrm{H}$ NMR spectrum of 1-(thiophen-3-yl)ethan-1-ol.

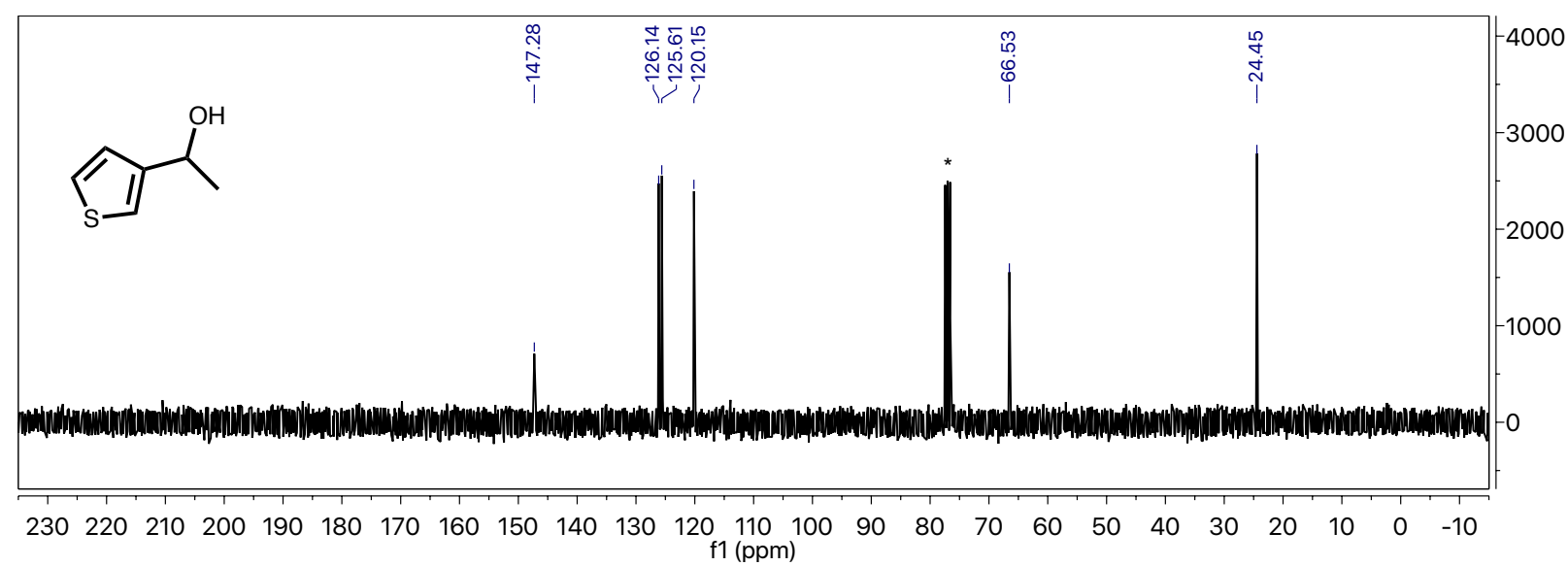

Figure S38. ${ }^{13} \mathrm{C}$ NMR spectrum of 1-(thiophen-3-yl)ethan-1-ol.

1-(pyridin-4-yl)ethan-1-ol (4dH2). Pale yellow oil (22 mg, 47 \% isolated yield). ${ }^{1} \mathrm{H}$ NMR (300 MHz, $\left.\mathrm{CDCl}_{3}\right) \delta 8.35(\mathrm{~d}, J=5.2 \mathrm{~Hz}, 2 \mathrm{H}), 7.25(\mathrm{~d}, J=5.2 \mathrm{~Hz}, 2 \mathrm{H}), 4.83(\mathrm{q}, J=6.6 \mathrm{~Hz}, 1 \mathrm{H}), 4.59(\mathrm{br} \mathrm{s}, 1 \mathrm{H}), 1.42(\mathrm{~d}, J=6.6$ $\mathrm{Hz}, 3 \mathrm{H}$ ). ${ }^{13 \mathrm{C} \mathrm{NMR}}\left(75 \mathrm{MHz}, \mathrm{CDCl}_{3}\right.$ ) $\delta=155.5,149.1,120.6,68.3$, 25.0. (+ denotes residual ethyl acetate).

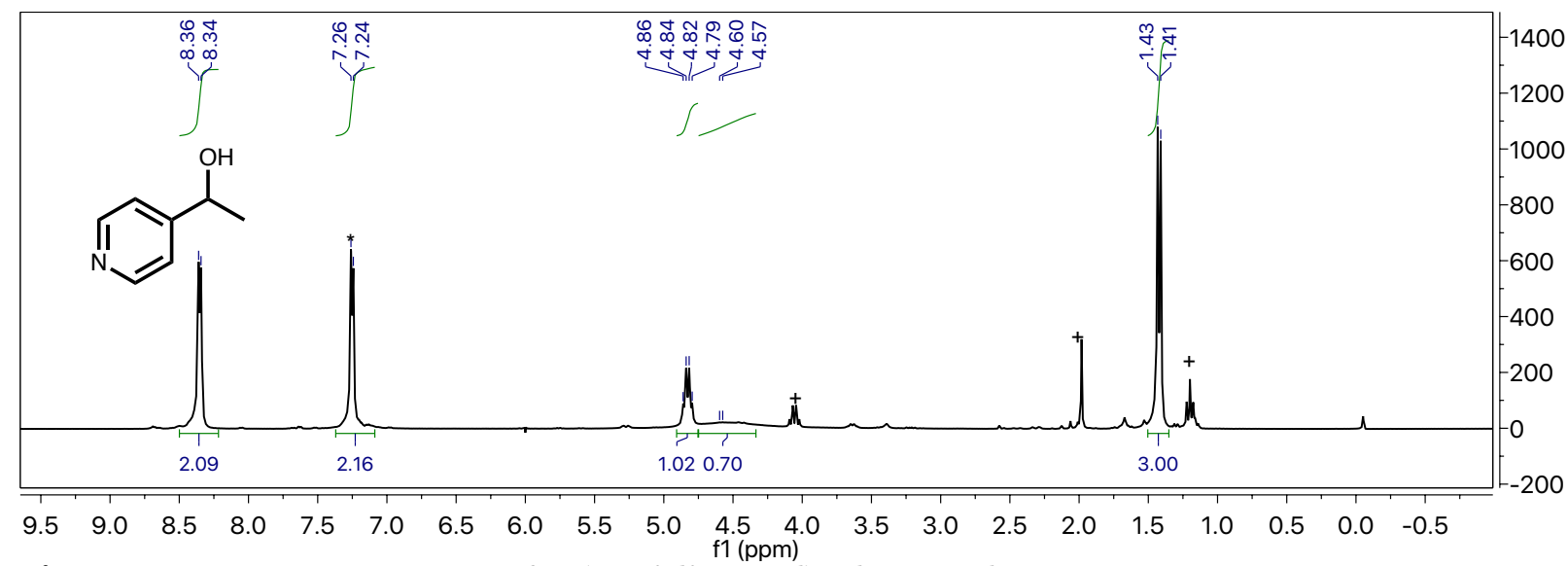

Figure S39. ${ }^{1} \mathrm{H}$ NMR spectrum of 1-(pyridin-4-yl)ethan-1-ol. 


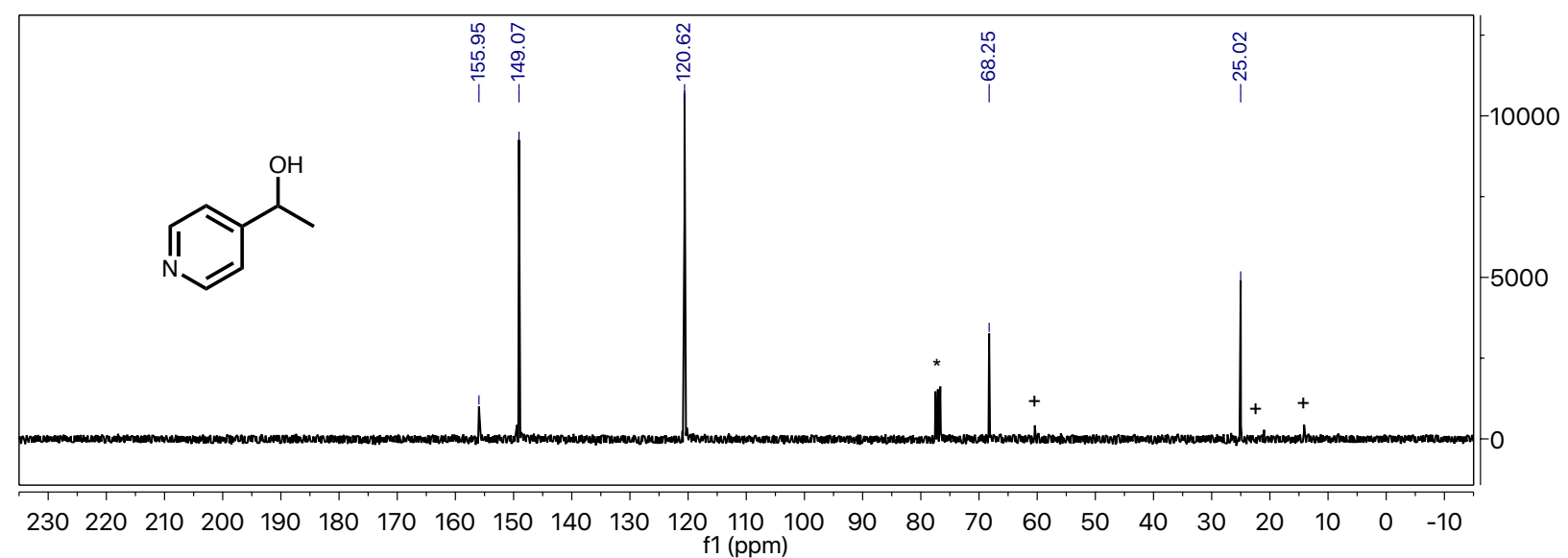

Figure S40. ${ }^{13} \mathrm{C}$ NMR spectrum of 1-(pyridin-4-yl)ethan-1-ol.

1,3-diphenylpropan-1-one (7aH2). Pale, yellow solid (68 mg, 84\% isolated yield) ${ }^{1} \mathrm{H}$ NMR (400 MHz, $\left.\mathrm{CDCl}_{3}\right) \delta 7.99(\mathrm{~d}, J=7.7 \mathrm{~Hz}, 2 \mathrm{H}), 7.45(\mathrm{t}, J=7.7 \mathrm{~Hz}, 2 \mathrm{H}), 7.35(\mathrm{~d}, J=4.3 \mathrm{~Hz}, 1 \mathrm{H}), 7.33-7.23(\mathrm{~m}, 4 \mathrm{H}), 7.22$ $-7.17(\mathrm{~m}, 1 \mathrm{H}), 3.3 \mathrm{O}(\mathrm{t}, J=7.7 \mathrm{~Hz}, 2 \mathrm{H}), 3.07$ (t, $J=7.7 \mathrm{~Hz}, 2 \mathrm{H}) .{ }^{13 \mathrm{C}} \mathrm{NMR}\left(75 \mathrm{MHz}, \mathrm{CDCl}_{3}\right) \delta=199.2,141.3$, 133.1, 128.6, 128.5, 128.4, 128.0, 128.1, 40.5, 30.1.

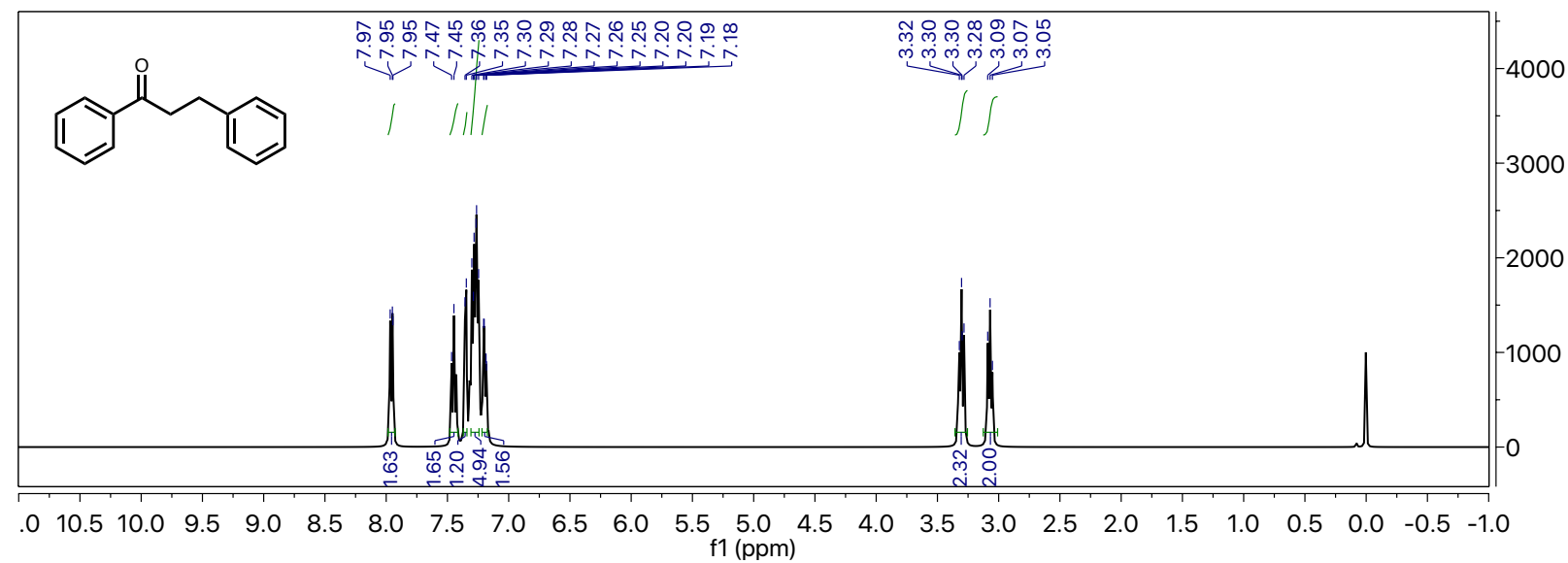

Figure S41. ${ }^{1} \mathrm{H}$ NMR spectrum of 1,3-diphenylpropan-1-one.

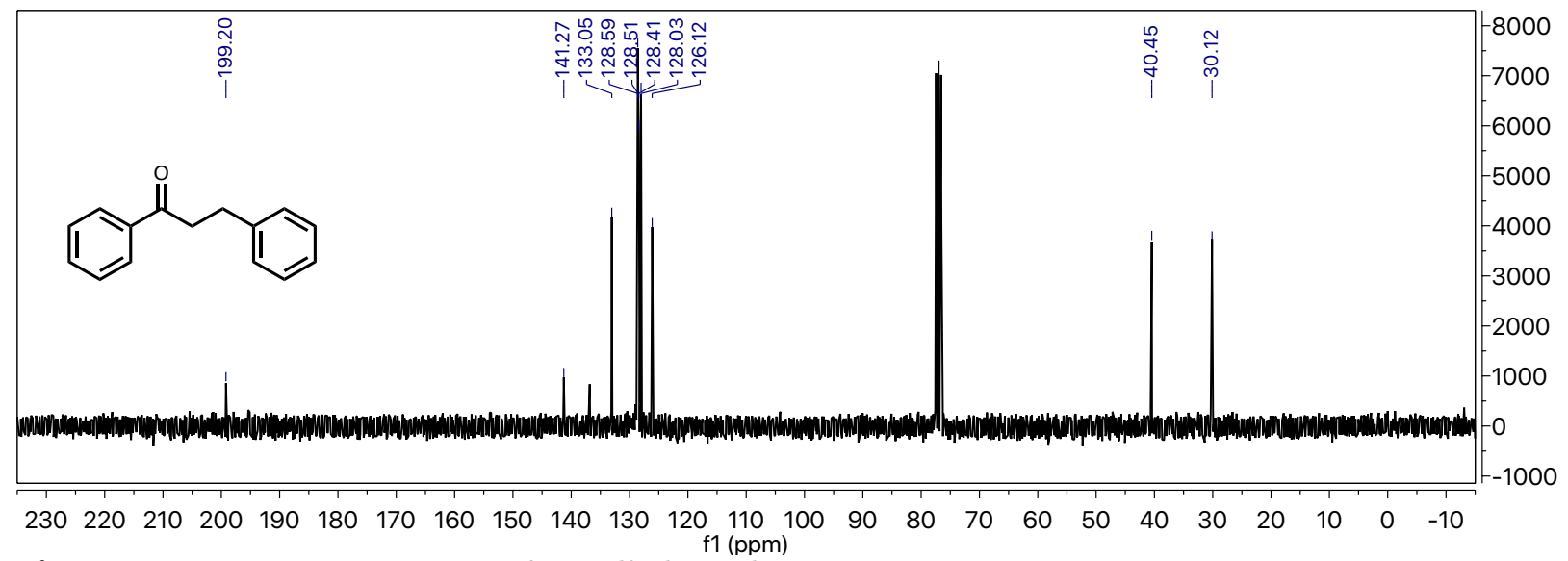

Figure S42. ${ }^{13} \mathrm{C}$ NMR spectrum of 1,3-diphenylpropan-1-one.

1-(4-methoxyphenyl)-3-phenylpropan-1-one (7a-OMe). Catalysis performed at higher scale (chalcone - $0.764 \mathrm{mmol}$ ) White, crystalline solid (178 mg, 97\% isolated yield). ${ }^{1} \mathrm{H} \mathrm{NMR}\left(300 \mathrm{MHz}, \mathrm{CDCl}_{3}\right)$ $\delta 7.95(\mathrm{~d}, J=8.8 \mathrm{~Hz}, 2 \mathrm{H}), 7.36-7.19(\mathrm{~m}, 5 \mathrm{H}), 6.92(\mathrm{~d}, J=8.7 \mathrm{~Hz}, 2 \mathrm{H}), 3.86(\mathrm{~s}, 3 \mathrm{H}), 3.25(\mathrm{dd}, J=8.6,6.7$ $\mathrm{Hz}, 2 \mathrm{H}), 3.06$ (t, $J=7.7 \mathrm{~Hz}, 2 \mathrm{H}) .{ }^{13 \mathrm{C}} \mathrm{NMR}\left(75 \mathrm{MHz}, \mathrm{CDCl}_{3}\right) \delta 197.8,163.4,141.5,130.3,128.5,128.4,126.1$, $113.7,55.4,40.1,30.3$. 


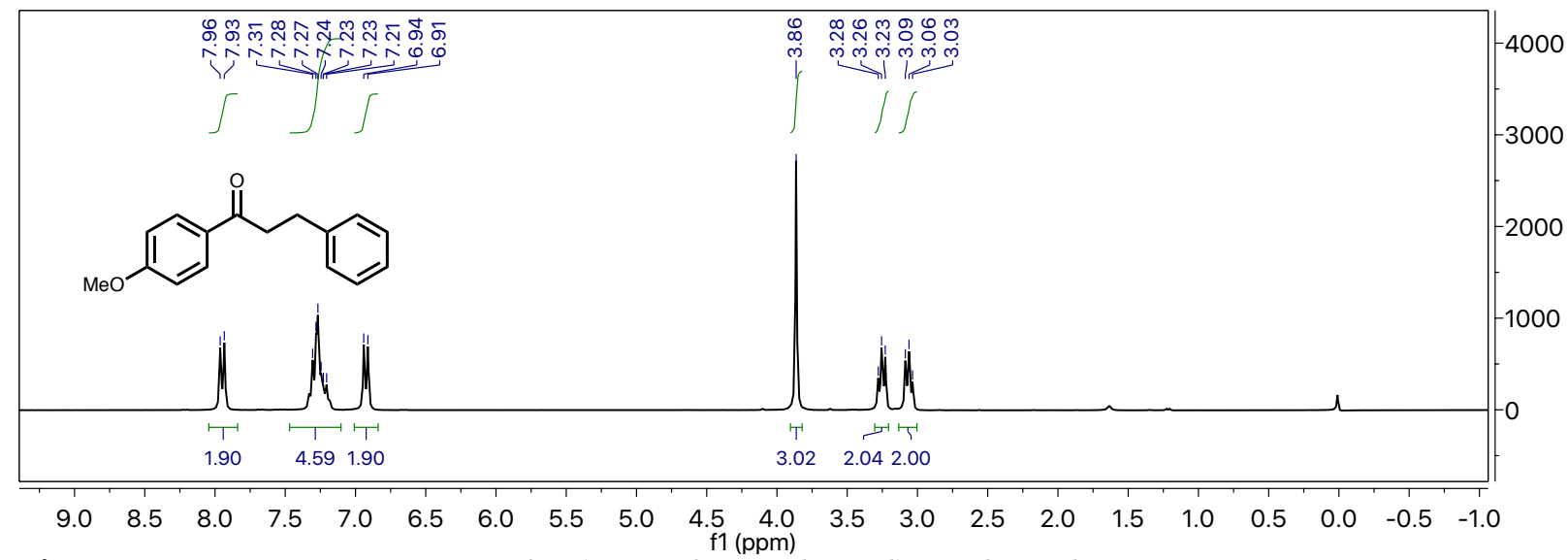

Figure S43. ${ }^{1 \mathrm{H}}$ NMR spectrum of 1-(4-methoxyphenyl)-3-phenylpropan-1-one.

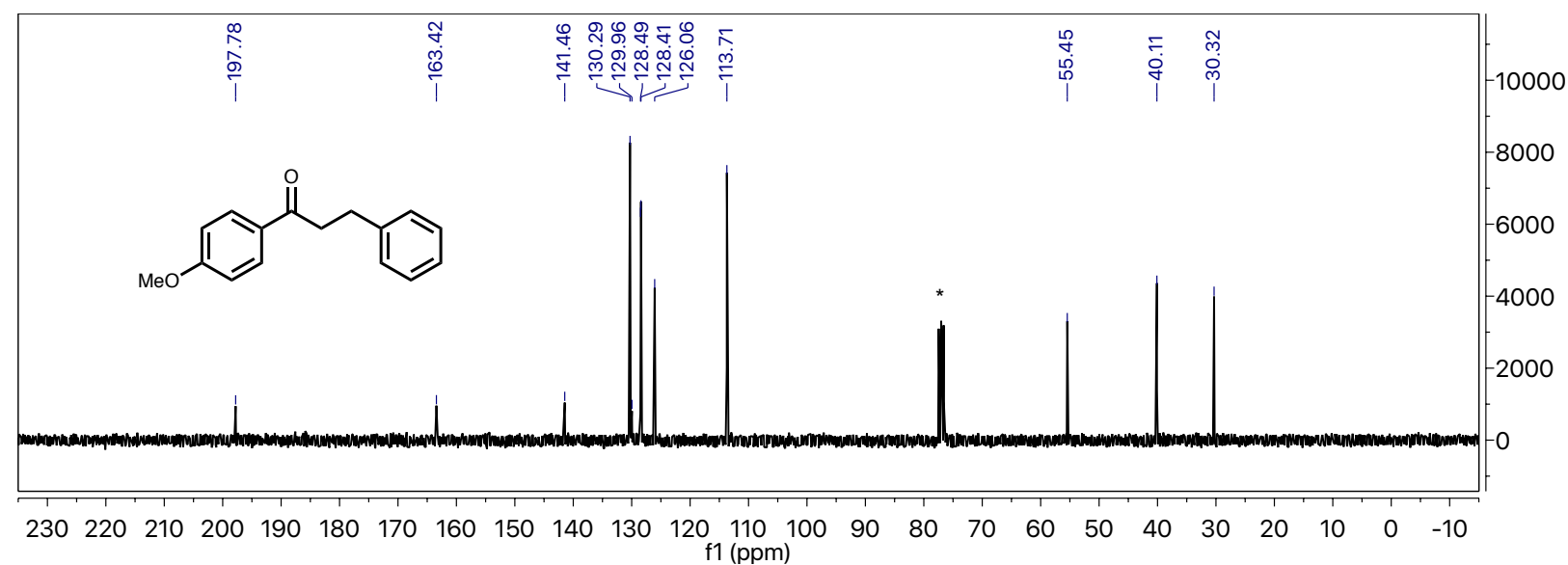

Figure S44. ${ }^{13 \mathrm{C}}$ NMR spectrum of 1-(4-methoxyphenyl)-3-phenylpropan-1-one.

3-phenyl-1-(p-tolyl)propan-1-one (7a-Me). Pale, yellow solid (74 $\mathrm{mg}, 78 \%$ isolated yield). ${ }^{1 \mathrm{H}} \mathrm{NMR}$ $\left(300 \mathrm{MHz}, \mathrm{CDCl}_{3}\right) \delta 7.86(\mathrm{~d}, J=8.2 \mathrm{~Hz}, 2 \mathrm{H}), 7.42-7.05(\mathrm{~m}, 7 \mathrm{H}), 3.27(\mathrm{dt}, J=6.6,4.4 \mathrm{~Hz}, 1 \mathrm{H}), 3.06(\mathrm{t}, J$ $=4.2 \mathrm{~Hz}, 1 \mathrm{H}), 2.40(\mathrm{~d}, J=2.5 \mathrm{~Hz}, 3 \mathrm{H})$.

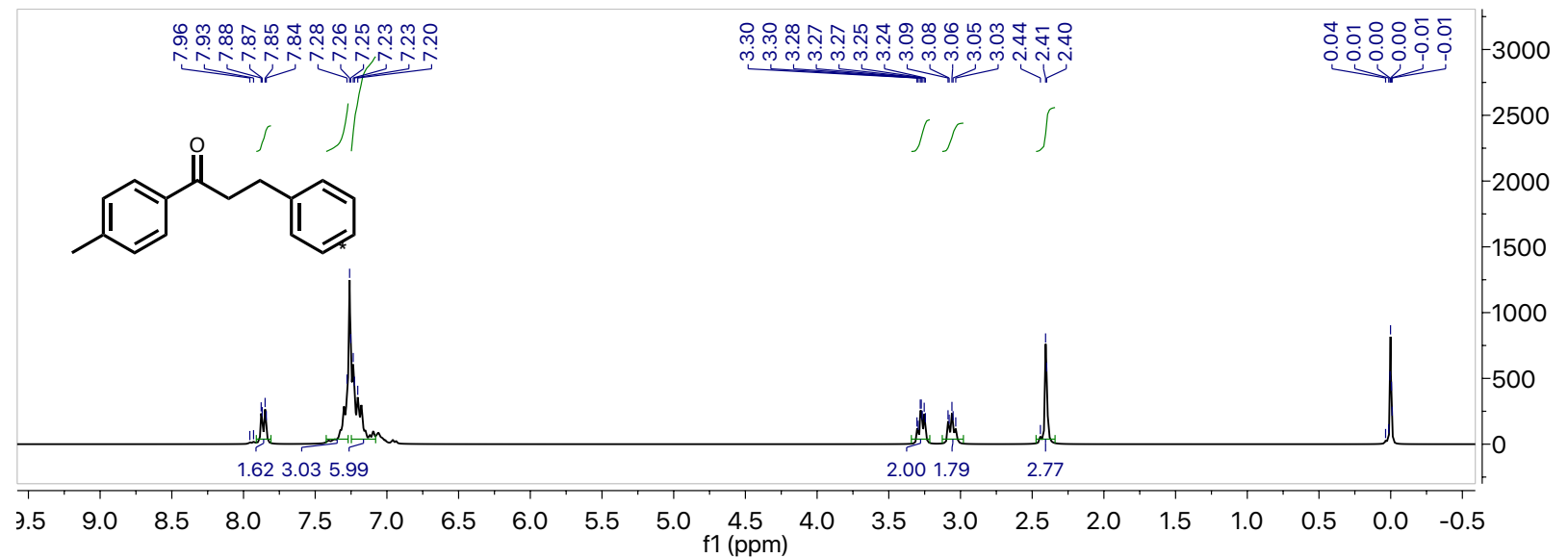

Figure S45. ${ }^{1} \mathrm{H}$ NMR spectrum of 3-phenyl-1-(p-tolyl)propan-1-one.

1,3-diphenylpropan-1-ol (7c-H). Pale yellow, solid (243 mg, 96\% isolated yield). ${ }^{1} \mathrm{H}$ NMR (300 MHz, $\left.\mathrm{CDCl}_{3}\right) \delta 7.38-7.16(\mathrm{~m}, 11 \mathrm{H}), 4.70(\mathrm{ddt}, J=8.4,3.7,2.0 \mathrm{~Hz}, 1 \mathrm{H}), 2.82-2.60(\mathrm{~m}, 3 \mathrm{H}), 2.21-1.96(\mathrm{~m}, 2 \mathrm{H})$, $1.83(\mathrm{~d}, J=3.4 \mathrm{~Hz}, 1 \mathrm{H})$. 


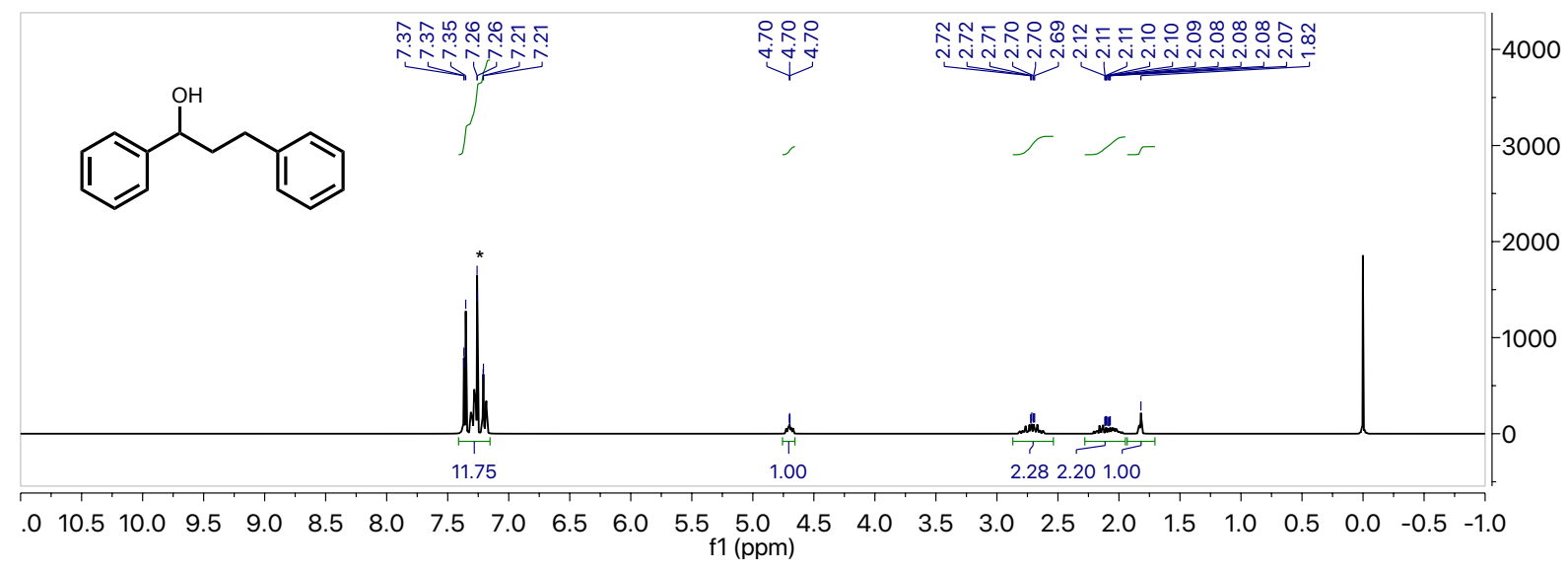

Figure S46. ${ }^{1} \mathrm{H}$ NMR spectrum of 1,3-diphenylpropan-1-ol.

2-benzyl-2,3-dihydro-1H-inden-1-one (9). Brown oil, solidifies on standing. (178 $\mathrm{mg}$, 70\% isolated yield, passed through silica plug with DCM:hexane (50:50) ${ }^{1} \mathrm{H}$ NMR $(300 \mathrm{MHz},) \delta 7.79(\mathrm{~d}, J=7.7 \mathrm{~Hz}, 1 \mathrm{H})$, $7.57(\mathrm{t}, J=7.5 \mathrm{~Hz}, 1 \mathrm{H}), 7.45-7.17(\mathrm{~m}, 7 \mathrm{H}), 3.41(\mathrm{dd}, J=14.0,4.1 \mathrm{~Hz}, 1 \mathrm{H}), 3.17(\mathrm{dd}, J=17.0,7.6 \mathrm{~Hz}, 1 \mathrm{H})$, 3.01 (ddt, $J=11.5,7.7,4.0 \mathrm{~Hz}, 1 \mathrm{H}), 2.86$ (dd, $J=17.0,3.9 \mathrm{~Hz}, 1 \mathrm{H}), 2.67$ (dd, $J=14.0,10.4 \mathrm{~Hz}, 1 \mathrm{H}$ ).. ${ }^{13 \mathrm{C}}$ $\operatorname{NMR}\left(75 \mathrm{MHz} \mathrm{CDCl}_{3}\right) \delta 207.8,153.6,139.6,136.5,134.8,128.9,128.5,127.4,126.6,126.3,124.0,48.9$, 37.0, 32.2. (+ denotes residual methylene chloride, $\dagger$ denotes silicone grease.)

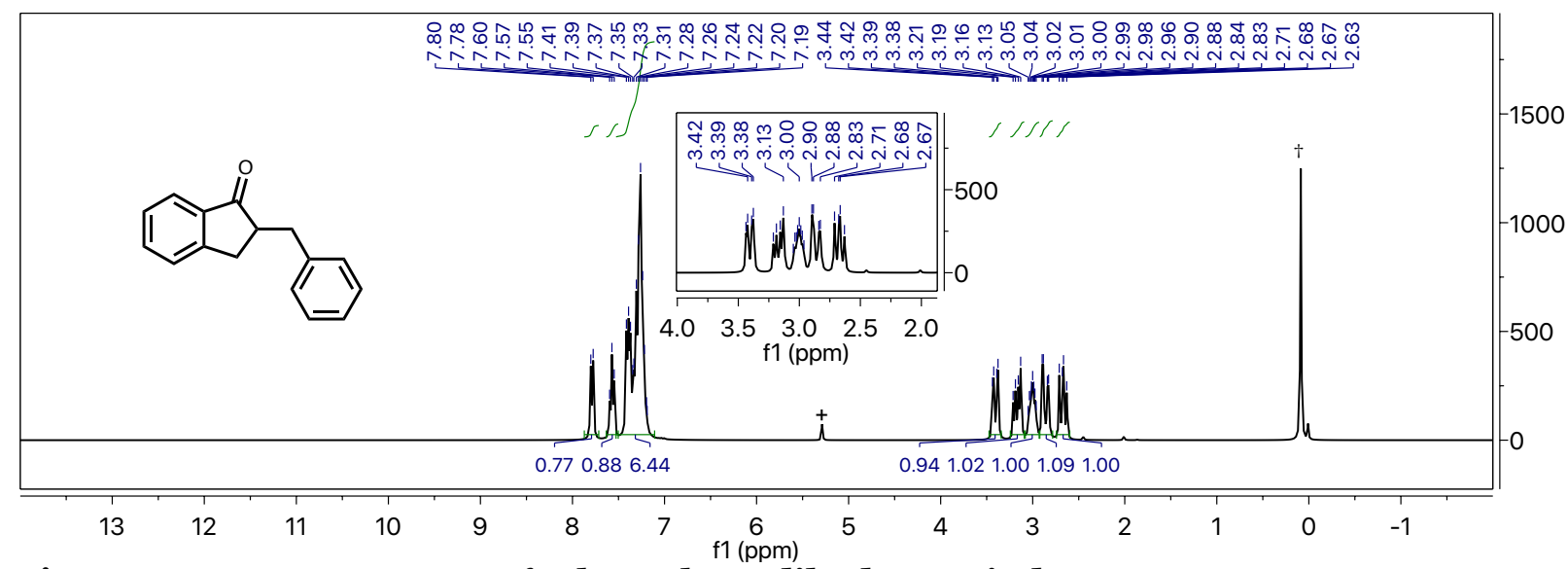

Figure S47. ${ }^{\mathrm{H}}$ NMR spectrum of 2-benzyl-2,3-dihydro-1H-inden-1-one.

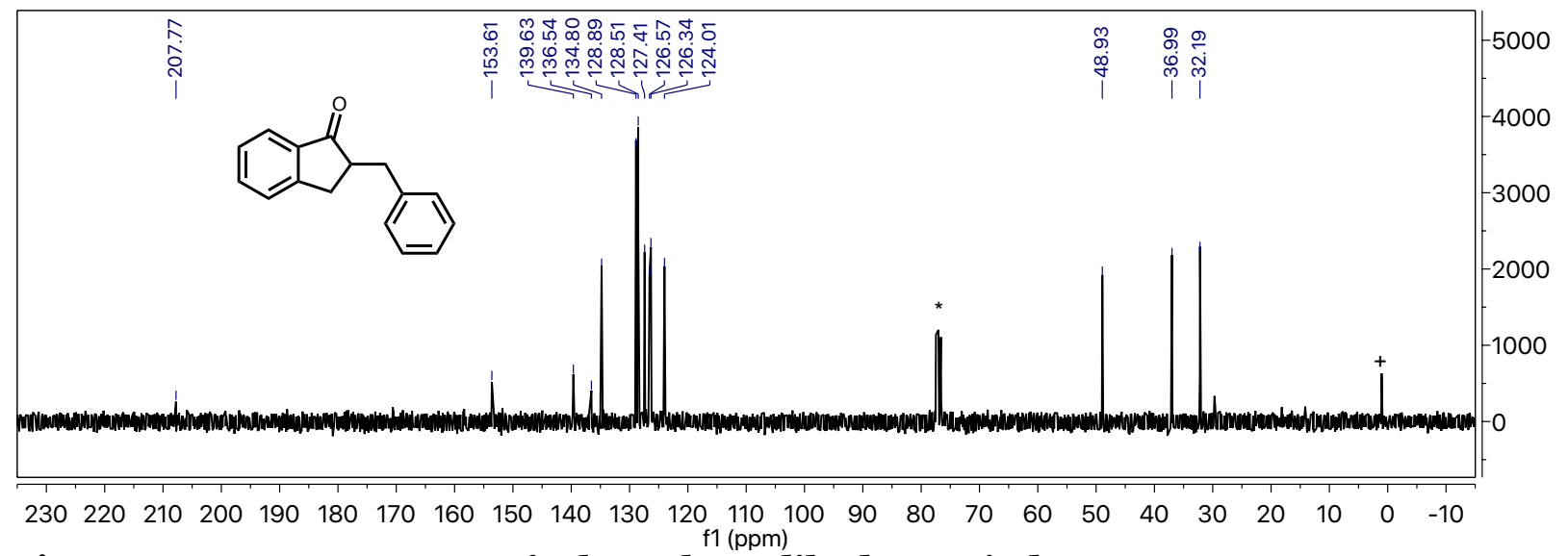

Figure S48. ${ }^{13} \mathrm{C}$ NMR spectrum of 2-benzyl-2,3-dihydro-1H-inden-1-one. 
4-phenylbutan-2-one (1Oa). Yellow, viscous oil ( $75 \mathrm{mg}, 100 \%$ isolated yield) ${ }^{1} \mathrm{H} \mathrm{NMR}$ (300 $\mathrm{MHz}, \mathrm{CDCl}_{3}$ ) $\delta 7.33-7.27(\mathrm{~m}, 1 \mathrm{H}), 7.24-7.14(\mathrm{~m}, 4 \mathrm{H}), 2.90(\mathrm{t}, J=7.6 \mathrm{~Hz}, 2 \mathrm{H}), 2.76(\mathrm{t}, J=7.7 \mathrm{~Hz}, 2 \mathrm{H}), 2.14(\mathrm{~s}, 3 \mathrm{H}) .{ }^{13 \mathrm{C}}$ NMR (75 MHz, $\mathrm{CDCl}_{3}$ ) $\delta$ 141.0, 129.0, 128.5, 128.4, 128.3, 126.1, 45.2, 29.7.

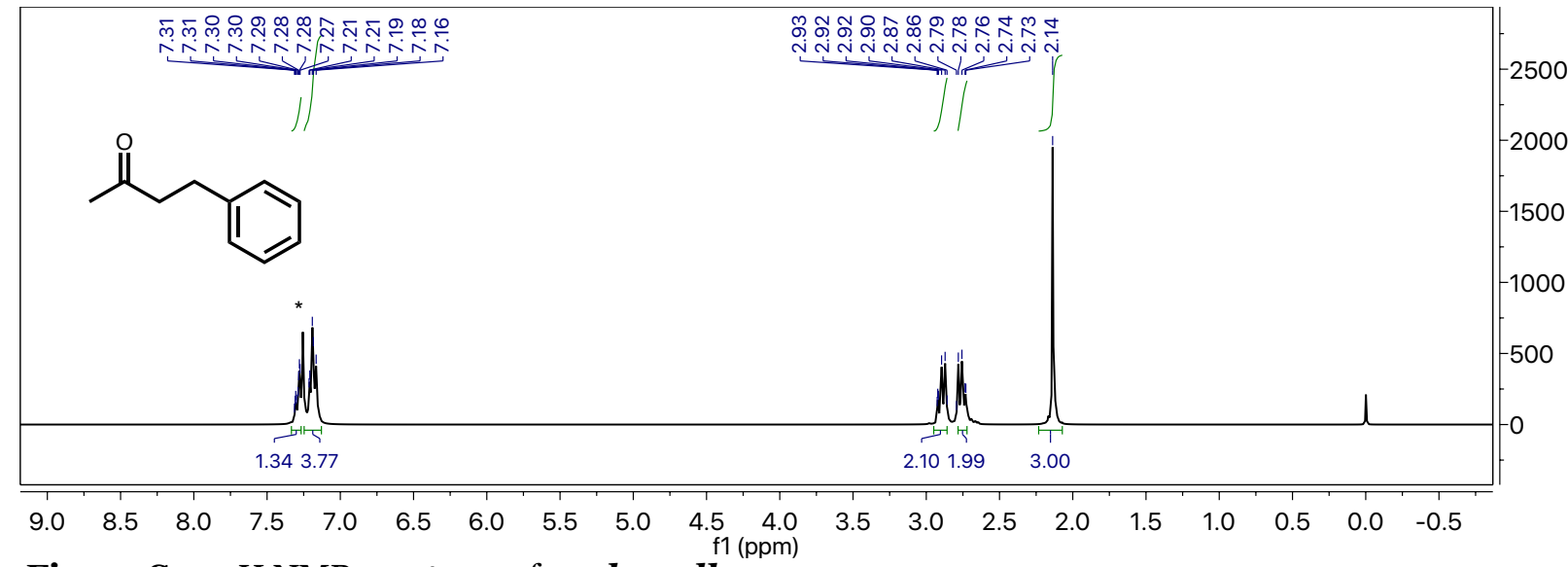

Figure S47. ${ }^{1} \mathrm{H}$ NMR spectrum of 4-phenylbutan-2-one.

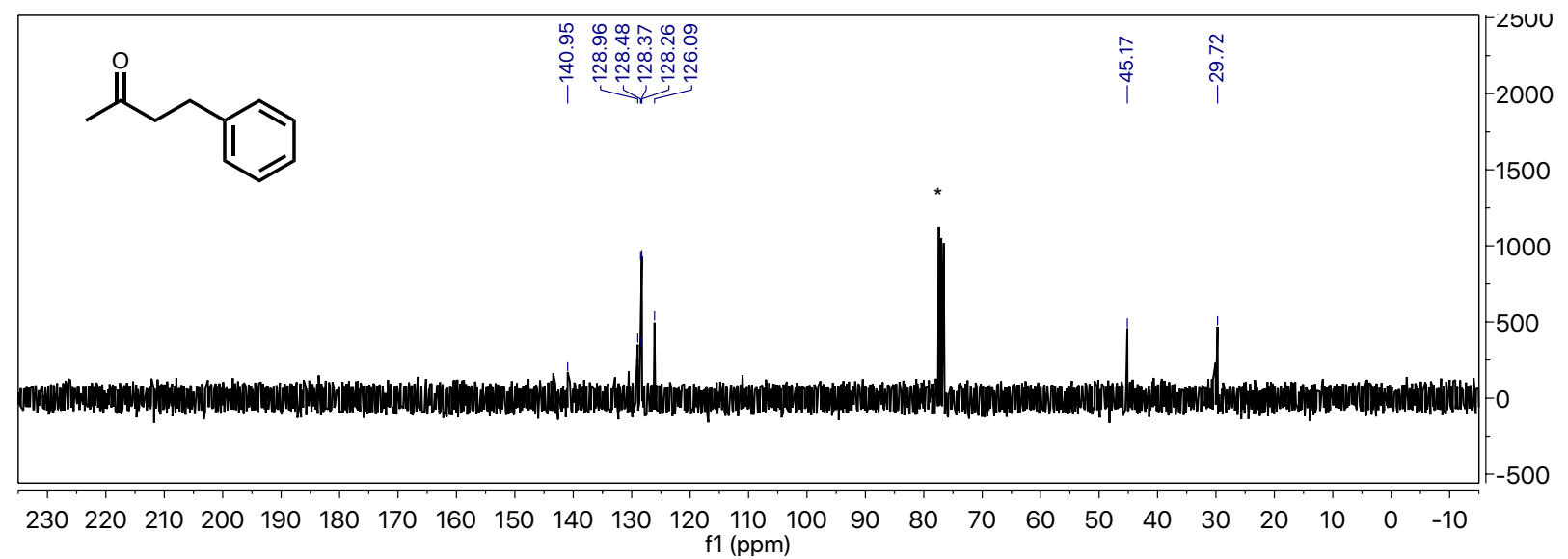

Figure S49. ${ }^{13 \mathrm{C}}$ NMR spectrum of 4-phenylbutan-2-one.

Pentan-2-one (11a). Clear, colorless oil (100\% conversion by NMR) ${ }^{1} \mathrm{H}$ NMR (300 MHz, toluene- $\left.d_{8}\right) \delta$ $1.99-1.79(\mathrm{~m}, 2 \mathrm{H}), 1.67(\mathrm{t}, J=5.1 \mathrm{~Hz}, 3 \mathrm{H}), 1.56-1.35(\mathrm{~m}, 2 \mathrm{H}), 0.79(\mathrm{dd}, J=7.4,4.7 \mathrm{~Hz}, 3 \mathrm{H}) .{ }^{13 \mathrm{C}} \mathrm{NMR}(75$ $\mathrm{MHz}$, toluene- $\left.d_{8}\right) \delta 205.3,44.8,28.7,17.0,13.3$.

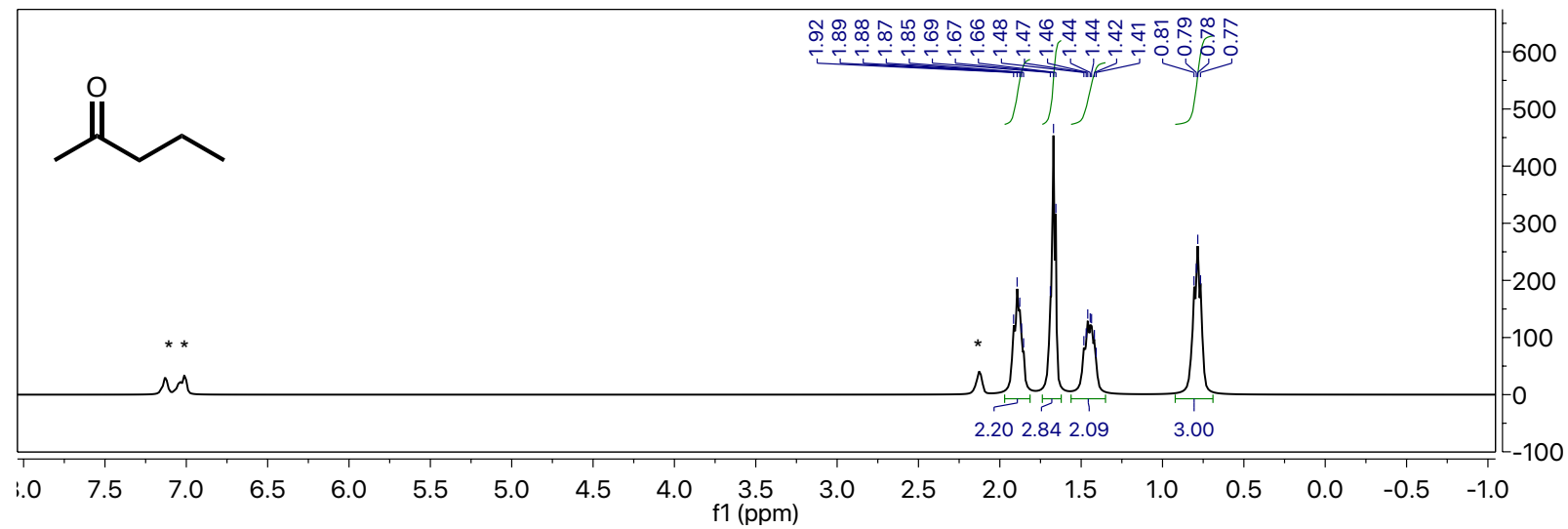

Figure S50. ${ }^{1} \mathrm{H}$ NMR spectrum of pentan-2-one. 


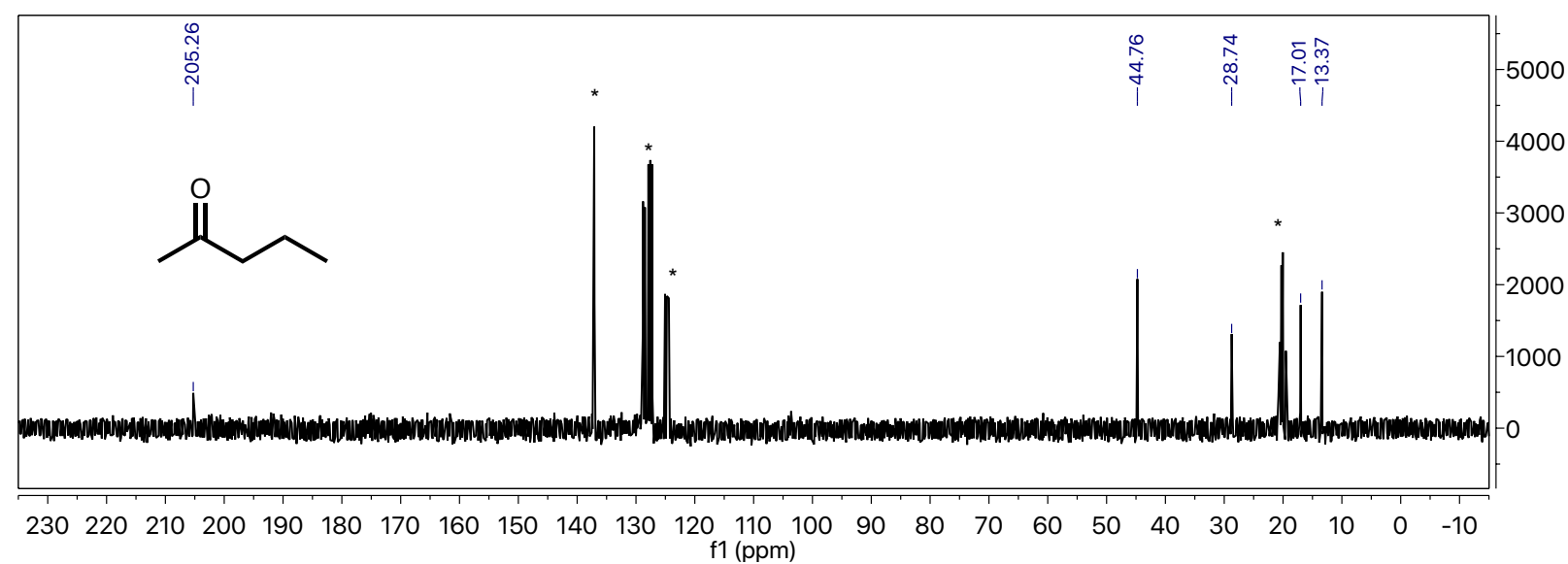

Figure S51. ${ }^{13 \mathrm{C}}$ NMR spectrum of pentan-2-one.

\section{References}

1. Chadwell, H. M.; Kohler, E. P. Org. Synth., 1922, 2, 1.

2. Luche, J-L.; Rodriguez-Hahn, L.; Crabbé, P. J. Chem. Soc. Chem. Commun. 1978, 601-602.

3. Testa, M. L.; Zaballos, E.; Zaragozá, R. J. Tetrahedron 2012, 68 (47), 9583-9591.

4. Edwards, P. G.; Fallis, I. A.; Yong, B. S. Brit. UK Pat. Appl. GB 2378182 A 2003

5. CrysAlisPro; Rigaku OD, The Woodlands, TX, 2015.

6. Sheldrick, G. M. Acta Cryst. 2015, $A 71,3-8$.

7. Sheldrick, G.M. Acta Cryst. 2008, A64, 112-122.

8. Müller, P. Crystallography Reviews 2009, 15, 57-83.

9. Ito, M.; Osaku, A.; Kobayashi, C.; Shiibashi, A.; Ikariya, T. Organometallics, 2009, 28 (2), 390-393. 10. van Putten, R.; Uslamin, E. A.; Garbe, M.; Liu, C.; Gonzalez-de-Castro, A.; Lutz, M.; Junge, K.;

Hensen, E. J. M.; Beller, M.; Lefort, L.; et al. Angew. Chemie Int. Ed. 2017, 56 (26), 7531-7534. 